\title{
OPTICAL EMISSION-LINE GAS ASSOCIATED WITH THE RADIO SOURCE 3C 277.3
}

\author{
WIL VAN BREUGEL ${ }^{1}$ \\ Radio Astronomy Laboratory, University of California, Berkeley \\ GeORGe MiLeY ${ }^{1}$ \\ Sterrewacht, Leiden, The Netherlands \\ TiMOTHY HeCKMAN ${ }^{1,2}$ \\ Astronomy Program, University of Maryland \\ HARVEY BUTCHER \\ Sterrenkundig Laboratorium "Kapteyn," Groningen, The Netherlands \\ AND \\ AlAN BRIDLE \\ National Radio Astronomy Observatory, Charlottesville, Virginia \\ Received 1984 April 25; accepted 1984 September 20
}

\begin{abstract}
We present the results of a detailed optical and radio investigation of the radio galaxy 3C 277.3. These data provide clear evidence for an interaction between the radio plasma and optical emission-line gas. First, narrow-band $\mathrm{H} \alpha$ images reveal a strong morphological relationship between the emission-line gas and the 60 $\mathrm{kpc}$ scale radio source. The brightest emission-line gas is found adjacent to the brightest radio features, and a faint shell-like $\mathrm{H} \alpha$ structure bounds much of the northern radio lobe. The southern radio jet bends sharply and then widens out at the location of a bright radio/optical knot (suggestive of a jet/cloud collision). Second, there is a good correlation between the presence of emission-line gas and the strong Faraday depolarization of the radio emission. Third, the emission-line kinematics are not those of quiescent material and appear to be influenced by the radio source. The emission lines are broader near the regions of strong radio emission; the gas shows a large (500-600 $\mathrm{km} \mathrm{s}^{-1}$ ) velocity change along the radio axis (compared with only $50-100 \mathrm{~km} \mathrm{~s}^{-1}$ in the perpendicular direction); a sharp velocity gradient is seen near the northern radio hot spot; the gas velocity field is very asymmetric relative to the galaxy nucleus. Fourth, the minimum-energy pressures in the southern radio knots are very close to the pressures derived independently for the associated emission-line gas $\left(\sim 10^{-9} \mathrm{dyn}^{-2}\right)$, suggesting that the two media are in rough pressure equilibrium.

We discuss our data in terms of twin radio jets which propagate through an inhomogeneous (cloudy) medium. We summarize evidence supporting a picture in which the emission-line gas is local material which is excited and accelerated by the radio source. First, the spectrum of the emission-line gas implies that it has significantly subsolar (Magellanic Cloud) abundances. This would be expected for ambient material located far $(\sim 10-40 \mathrm{kpc})$ from the galaxy nucleus. Second, the morphologies of the southern half of the radio source and the emission-line region strongly suggest that the radio jet has collided with a massive $\left(2 \times 10^{6} M_{\odot}\right.$ of emission-line gas is detected) ambient cloud. Study of other radio sources with associated emission-line gas also supports such a picture. We hypothesize that the collision between the southern jet and the cloud causes the radio emission to flare up, produces an ionizing synchrotron continuum (the optical/infrared counterpart of which has been directly detected), and deflects and entrains the jet material. We argue that the Faraday depolarization seen throughout regions of emission-line gas is produced by clumpy thermal material in the boundary layers of the radio source (a Faraday screen model). The peculiar polarization morphology of the southern lobe (large depolarization along the jet direction and a trough in the percentage polarization and total intensity which bisects the lobe) is attributed to the continuous supply of entrained ionized gas by the jet. We investigate whether ambient material which has been shocked by the outbound radio plasma at the northern hot spot can cool rapidly enough to explain the proximity of the radio and emission-line regions. Existing limits to the density of a putative gaseous halo around 3C 277.3 (from X-ray data) are stringent enough to rule out a uniform (single-phase) halo as the source of the emission-line gas. Our data then require that the halo is cloudy (cloud densities of $0.1-1 \mathrm{~cm}^{-3}$ and a total mass of $10^{7}-10^{8} M_{\odot}$ for the cloud system). We emphasize that in our picture, the observed gas velocities are not those of the jet material but are indirectly related to the expansion and translation velocities of boundaries of the radio source.
\end{abstract}

Subject headings: galaxies: individual — radio sources: galaxies

\footnotetext{
${ }^{1}$ Visiting Astronomer, National Radio Astronomy Observatory, and Visiting Astronomer, Kitt Peak National Observatory.

2 Alfred P. Sloan Foundation Fellow.
} 
TABLE 1

GLOBAL Properties OF 3C 277.3

\begin{tabular}{|c|c|c|}
\hline Parameter & Value & Ref. \\
\hline \multicolumn{3}{|l|}{ Radio } \\
\hline Flux density $(1400 \mathrm{MHz}) \ldots \ldots \ldots \ldots$ & $3.0 \mathrm{Jy}$ & 1 \\
\hline Monochromatic power $(1400 \mathrm{MHz})$.. & $4.3 \times 10^{25} \mathrm{~W} \mathrm{~Hz}^{-1}$ & 1 \\
\hline Total radio luminosity $\left(10^{7}-10^{11} \mathrm{~Hz}\right)$. & $6.7 \times 10^{35} \mathrm{~W}$ & 1 \\
\hline Spectral index $\left(S_{v} \propto v^{-\alpha}\right) \ldots \ldots \ldots \ldots \ldots$ & 0.7 & 1 \\
\hline \multicolumn{3}{|l|}{ Optical } \\
\hline 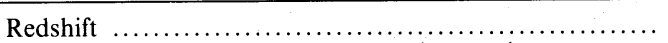 & 0.0857 & 2 \\
\hline Luminosity distance $\left(H_{0}=75 \mathrm{~km} \mathrm{~s}^{-1} \mathrm{Mpc}^{-1}\right)$ & $350 \mathrm{Mpc}$ & 2 \\
\hline 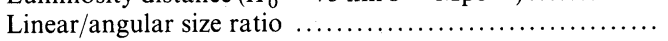 & $1.45 \mathrm{kpc} \operatorname{arcsec}^{-1}$ & 2 \\
\hline 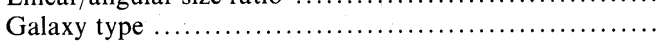 & E0 & 3 \\
\hline Absolute visual magnitude ............. & -21.8 & 4 \\
\hline
\end{tabular}

REFERENCES.-11) Kellermann, Pauliny-Toth, and Williams 1969. (2) Schmidt 1965. (3) This paper; Griffin 1963. (4) Burbidge and Crowne 1979.

\section{INTRODUCTION}

In a previous paper (Miley et al. 1981) we reported the discovery of spatially extended optical line and continuum emission associated with $3 \mathrm{C} 277.3$, a radio galaxy at a redshift of 0.0857 (Bridle et al. 1981). In particular we concluded that the brightness of the optical line emission was correlated with the total intensity of the radio continuum and anticorrelated with its polarized intensity. It was further shown that the lineemitting gas had velocities of up to a few hundred kilometers per second with respect to the nucleus and was redshifted near the northern hot spot and blueshifted near the southern jet. These conclusions concerning the emission-line gas were drawn from spectroscopic results obtained using a single-slit position angle.

This paper reports new radio and optical information. First, we have used the NRAO Very Large Array (VLA) to obtain radio maps at several wavelengths with a resolution similar to that of our optical data. Second, using a CCD on the $4 \mathrm{~m}$ telescope at Kitt Peak National Observatory, we have obtained narrow-band images in sub-arcsecond seeing which show the detailed morphology of the line-emitting gas. Third, using long slit spectroscopes on the $4 \mathrm{~m}$ telescope, we have obtained kinematic information about the gas at various posi- tions and in several position angles. Fourth, using an aperture spectroscope on the 90 inch $(2.3 \mathrm{~m})$ telescope of Steward Observatory, we have observed the southern jet in order to study the density and temperature of the line-emitting gas.

These radio and optical data demonstrate conclusively the existence of morphological and energetic relationships between the radio plasma and the emission-line gas. In $\S$ II we describe the various observations. The results are presented in $\S$ III and discussed in $\S$ IV. Some global properties of 3C 277.3 are given in Table 1.

\section{OBSERVATIONS AND REDUCTION}

Tables 2 and 3 summarize parameters pertaining to the radio and optical observations, respectively.

\section{a) Radio}

3C 277.3 was observed at $1.4,4.9$, and $15 \mathrm{GHz}(21,6$, and 2 $\mathrm{cm}$ wavelengths) using the VLA (Thompson et al. 1980) in its $\mathrm{A}, \mathrm{B}$, and $\mathrm{C}$ configurations, respectively. The $(u, v)$-coverage is similar at each of these wavelengths; so that maps at 1 .'2 resolution can be compared directly without bias due to differing sensitivities on different structural scales or due to differing sidelobe structures.

TABLE 2

A. Log of the Radio Observations ${ }^{\mathrm{a}}$

\begin{tabular}{|c|c|c|c|c|c|c|}
\hline VLA Configuration $^{\mathrm{b}}$ & Date & $\begin{array}{c}\text { Frequency } \\
(\mathrm{MHz})\end{array}$ & $\begin{array}{l}\text { Wavelength } \\
\quad(\mathrm{cm})\end{array}$ & $\begin{array}{c}\text { Bandwidth } \\
(\mathrm{MHz})\end{array}$ & $\begin{array}{l}\text { Resolution } \\
\quad(\operatorname{arcsec})\end{array}$ & $\begin{array}{c}\text { Observing } \\
\text { Time } \\
\text { (hr) }\end{array}$ \\
\hline 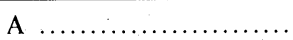 & $1981 \mathrm{Mar}$ & 4873 & 6.2 & 25 & 0.4 & 4 \\
\hline 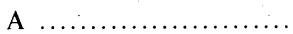 & $1981 \mathrm{Mar}$ & 1413 & 21.2 & 25 & 1.2 & 4 \\
\hline ….................. & 1981 Jun & 4885 & 6.1 & 50 & 1.2 & 8 \\
\hline 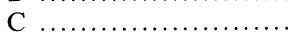 & 1981 Nov & 14965 & 2.0 & 50 & 1.2 & 8 \\
\hline
\end{tabular}

B. Primary Flux Density and Polarization Calibrator: 3C 286

\begin{tabular}{ccc}
\hline \hline Frequency & $\begin{array}{c}\text { Assumed } \\
\text { Flux Density } \\
(\mathrm{MHz})\end{array}$ & $\begin{array}{c}\text { Assumed Polarization } \\
\text { Position Angle } \\
\text { (degrees) }\end{array}$ \\
\hline $1413 \ldots \ldots$ & 14.77 & 33 \\
$4880 \ldots \ldots$ & 7.41 & 33 \\
$14965 \ldots \ldots$ & 3.44 & 33 \\
\hline
\end{tabular}

a Pointing position: R.A. $(1950)=12^{\mathrm{h}} 53^{\mathrm{m}} 18^{\mathrm{s}}$, decl. $(1950)=27^{\circ} 43^{\prime} 37^{\prime \prime}$.

b Thompson et al. 1980 . 
TABLE 3

Parameters of the Optical Observations

\begin{tabular}{|c|c|c|c|c|}
\hline Parameter & Imaging & Spectroscopy & Spectroscopy & Spectroscopy \\
\hline Telescope $\ldots \ldots \ldots \ldots \ldots \ldots \ldots$ & $4 \mathrm{~m}, \mathrm{KPNO}$ & $4 \mathrm{~m}, \mathrm{KPNO}$ & $4 \mathrm{~m}, \mathrm{KPNO}$ & $2.3 \mathrm{~m}$, Steward Obs. \\
\hline Auxiliary instrument...$\ldots \ldots \ldots$ & prime-focus $\mathrm{CCD}$ & Cryogenic Camera & HGVS & red Reticon \\
\hline Wavelength coverage $\ldots \ldots \ldots \ldots$ & $75 \AA$ at $\mathrm{H} \alpha$ & $4500-7900 \AA$ & $5110-5800 \AA$ & $4000-8000 \AA$ \\
\hline Seeing $\ldots \ldots \ldots \ldots \ldots \ldots \ldots \ldots \ldots$ & $\sim 0.8$ & $\sim 2^{\prime \prime}$ & $2^{\prime \prime}-3^{\prime \prime}$ & $\ldots$ \\
\hline Field size $\ldots \ldots \ldots \ldots \ldots \ldots \ldots \ldots$ & $112^{\prime \prime} \times 85^{\prime \prime}$ & $3^{\prime \prime} .2 \times 300^{\prime \prime}$ & $2^{\prime \prime} \times 166^{\prime \prime}$ & $4^{\prime \prime}$ circular \\
\hline Pixel size $\ldots \ldots \ldots \ldots \ldots \ldots \ldots \ldots$ & $0^{\prime \prime} .59 \times 0^{\prime \prime} .35$ & $0 " 8 \times 4.3 \AA$ & $1 " 3 \times 1.35 \AA$ & $2 \AA$ \\
\hline Number of pixels...$\ldots \ldots \ldots \ldots$ & $190 \times 244$ & $500 \times 800$ & $128 \times 512$ & 2048 \\
\hline Position angles $\ldots \ldots \ldots \ldots \ldots \ldots$ & $\ldots$ & $\begin{array}{l}\text { a. } 155^{\circ}, \text { through nucleus } \\
\text { b. } 155^{\circ}, 10^{\prime \prime} \mathrm{W} \text { of nucleus }\end{array}$ & $\begin{array}{l}\text { a. } 69^{\circ} \text {, through knot } \mathrm{K}_{1} \\
\text { b. } 77^{\circ}, 9^{\prime \prime} 2 \mathrm{~W} \text { and } 17^{\prime \prime} 6 \mathrm{~N} \text { of nucleus }\end{array}$ & centered on radio knot $K_{1}$ \\
\hline Date $\ldots \ldots \ldots \ldots \ldots \ldots \ldots \ldots \ldots \ldots$ & 1982 Jan & 1981 Nov & 1982 Apr & 1981 Jan \\
\hline Purpose of observations $\ldots \ldots \ldots$ & morphology of gas & $\begin{array}{l}\text { kinematics, ionization state, } \\
\text { density of gas }\end{array}$ & kinematics of gas & $\begin{array}{l}\text { gas density, temperature, } \\
\text { reddening }\end{array}$ \\
\hline
\end{tabular}

The source was also observed at $6 \mathrm{~cm}$ in the A configuration, yielding maps at 0 " 4 resolution. All maps have been CLEANed and restored with truncated circular Gaussian beams.

For all observations, 3C 286 served as the primary flux density and polarization position angle calibrator (Table 2). The secondary calibrator was $3 \mathrm{C} 287$, which was used to monitor amplitude and phase fluctuations of both instrumental and atmospheric origin and to calibrate the instrumental polarization. The data were reduced using the NRAO Astronomical Image Processing System (AIPS). Particular care was taken to match the projected baseline $(u, v)$-coverage and tapering of the data from the various frequencies before performing the Fourier transforms. All maps were self-calibrated to maximize their dynamic range.

\section{b) Optical \\ i) Imaging}

To map the optical line emission $(\mathrm{H} \alpha+[\mathrm{N}$ II $])$ several narrow-band ( $\sim 75 \AA$ full width at half-maximum [FWHM]) exposures were taken centered on the redshifted $\mathrm{H} \alpha$ line with a prototype Fairchild $211 \mathrm{CCD}$ (see, for example, Monet and Dahn 1983) at the prime focus of the 4 m telescope at Kitt Peak National Observatory (KPNO). Corresponding "off-band" exposures were taken centered in wavelength on the adjacent continuum. The CCD used in these observations has a relatively thick chip. While the interference properties of this chip made it excellent for narrow-band imaging, the CCD was also sensitive to cosmic-ray events. To overcome this problem four separate exposures were taken in each band. The four image frames were then combined by taking the median of each pixel value. Use of the median rejects extreme values and thereby removes cosmic-ray events (which are unlikely to occur at the same pixel on more than one of the four exposures). The onand off-band images were then flat-fielded, aligned, corrected for zero-level offsets due to the sky, and scaled to account for differences in filter throughput. The $\mathrm{H} \alpha+[\mathrm{N}$ II $]$ image was then obtained by subtracting the off-band from the on-band. The seeing during the observations was well below 1", resulting in a slight undersampling of the image in right ascension (pixel sizes 0 ".59 and 0".35 in R.A. and decl., respectively). We therefore smoothed the final image using circular Gaussian beams with 0 . 8 and 1 ." 5 resolution (Fig. 4).

The alignment between the orientation of the columns in the optical frame and true north on the sky is better than $1^{\circ}$. This was confirmed through comparisons with other optical images obtained with the videocamera on the $4 \mathrm{~m}$ (Miley et al. 1981) and $2.1 \mathrm{~m}$ (unpublished results) telescopes of KPNO. ii) Spectroscopy

To obtain information on the properties and kinematics of the line-emitting gas, long slit spectra were taken with the High Gain Video Spectrometer (HGVS) and Cryogenic Camera in conjunction with the Ritchey-Chretien spectrograph on the KPNO $4 \mathrm{~m}$ telescope. The CCD-based Cryogenic Camera is more sensitive than the HGVS except for wavelengths below $\sim 4500 \AA$, whereas the HGVS has the advantage of better wavelength resolution. We also observed the bright region of emission-line gas associated with the southern radio jet using the analog red Reticon spectroscopic system on the $2.3 \mathrm{~m}$ telescope of the Steward Observatory. These one-dimensional data have better signal-to-noise ratio in the red than the HGVS data and better spectral resolution $(\sim 6 \AA)$ than the Cryogenic Camera data. For all of these detector/telescope combinations, the data were obtained and flat-fielded in the standard way (e.g., Heckman et al. 1982; Balick and Heckman 1982). Details concerning the various instrumental parameters and positions covered are given in Table 3.

The spectroscopic data have been calibrated in terms of intensity versus wavelength with reference to the nucleus of the parent galaxy of $3 \mathrm{C} 277.3$, for which published absolute spectrophotometry exists (Yee and Oke 1978). However, because the spatial and spectroscopic resolutions of the Cryogenic Camera vary across the detector (see Balick and Heckman 1982), it is difficult to place these data on an accurate spectrophotometric scale. Hence, it is not possible to tie together the red (Cryogenic Camera) and the blue (HGVS) data accurately in an absolute sense. We have determined the relative intensities of prominent emission lines at various locations in $3 \mathrm{C}$ 277.3 assuming that $\mathrm{H} \alpha / \mathrm{H} \beta=3$ in regions other than the jet (where we do have spectrophotometric data from the $2.3 \mathrm{~m}$ telescope). This is consistent with the value implied by the (inaccurately linked) HGVS and Cryogenic Camera data and corresponds to a case $\mathrm{B}$ spectrum with negligible dust reddening.

\section{RESULTS}

$$
\begin{aligned}
& \text { a) Radio } \\
& \text { i) Morphology }
\end{aligned}
$$

The large- and small-scale structure of 3C 277.3 is shown in Figure $1\left(21 \mathrm{~cm}, 1^{\prime \prime} 2\right)$ and $2(6 \mathrm{~cm}, 0.4)$. At $2 \mathrm{~cm}$, only the brightest components of the source were detected (hot spot, nucleus, knots), so no map.is given. 3C 277.3 has a typical radio morphology for its luminosity in the $10 \mathrm{MHz}-100 \mathrm{GHz}$ range 


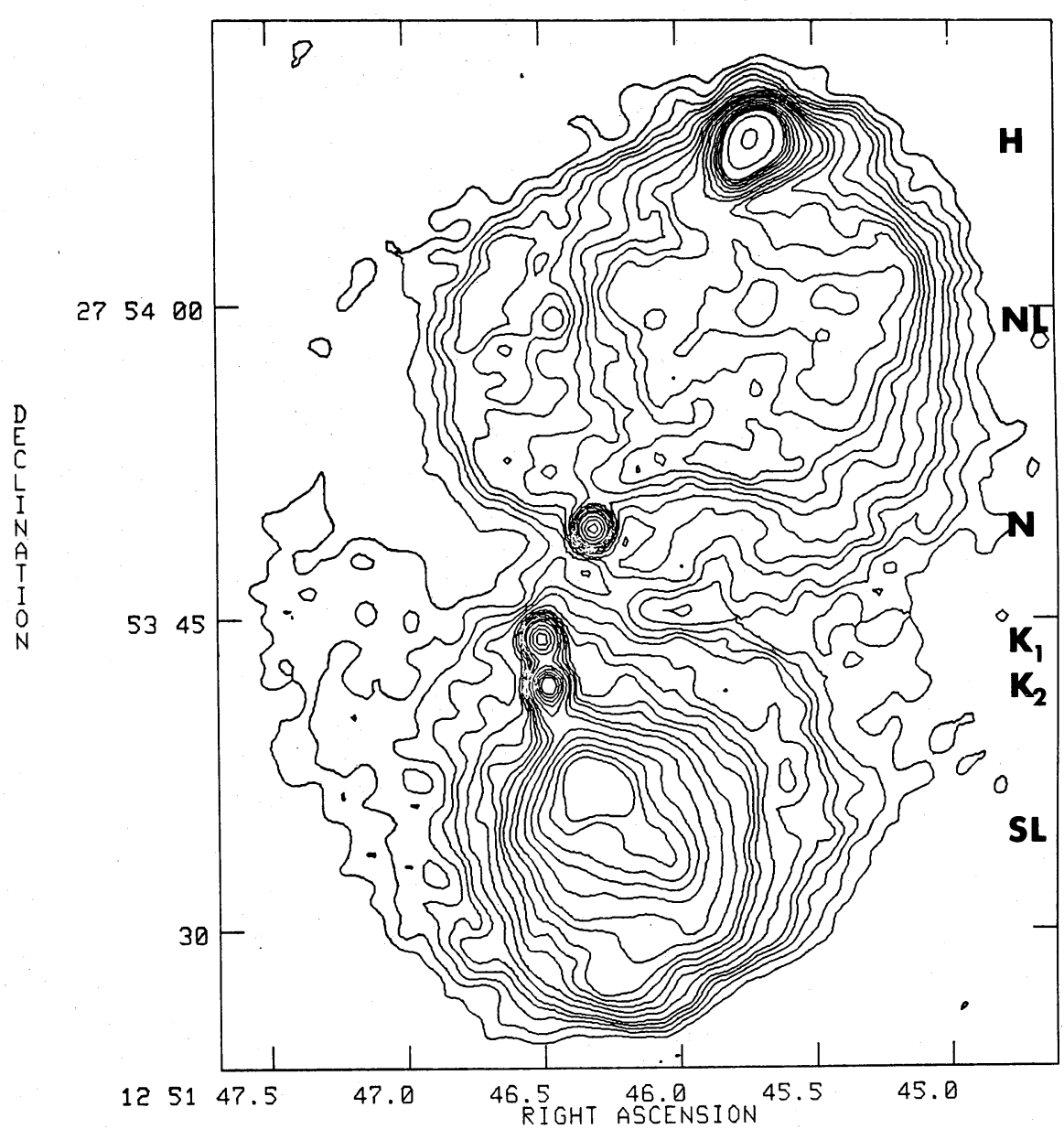

FIG. 1.-Contour representation of the total intensity of $3 \mathrm{C} 277.3$ at $21.2 \mathrm{~cm}\left(11^{\prime \prime} 2 \times 1\right.$."2 resolution). Various features are labeled: the northern hot spot (H), the northern lobe $(\mathrm{NL})$, the nucleus $(\mathrm{N})$, the two knots in the jet $\left(\mathrm{K}_{1}, \mathrm{~K}_{2}\right)$, and the southern lobe $(\mathrm{SL})$. Contour values (in $\mathrm{mJy}$ per beam) are $0.57 \times(-0.9,0.9,1.5,2.0,2.5$, $3,4,5,6,7,8,10,12,16,20,24,28,32,50,90)$. Negative values would have appeared dashed.

$\left(7 \times 10^{35} \mathrm{~W}\right.$ assuming a Hubble constant of $H_{0}=75 \mathrm{~km} \mathrm{~s}^{-1}$ $\mathrm{Mpc}^{-1}$ and $q_{0}=0.5$ ): wide, diffuse lobes with a few brightness enhancements (southern jet and northern hot spot). Some features are particularly noteworthy:

1. Both the northern and the southern halves of the source suggest the presence of relatively bright lobes within larger, more diffuse emission.

2. The northern (but not the southern) lobe has a prominent resolved hot spot $(\mathrm{H})$ at its outer edge. The interior of this lobe has clumpy structure.

3. The axis defined by the two bright southern jet knots, $\mathrm{K}_{1}$ and $\mathrm{K}_{2}$, is misaligned by $\sim 30^{\circ}$ with respect to the "fundamental" radio axis (defined by the northern hot spot $[\mathrm{H}]$, the nucleus $[\mathrm{N}]$, and the first jet knot $\left[\mathrm{K}_{1}\right]$ ). Unlike the northern lobe, the entire southern lobe appears bent.

4. The two knots $\left(K_{1}\right.$ and $\left.K_{2}\right)$ have their steepest intensity gradients on their "upstream" (northeastern) sides. This is clearly seen in the 0 ". 4 resolution $4.9 \mathrm{GHz}$ data shown in Figure 4, panel 4.

5. The jet emission flares out with an opening angle of $\sim 40^{\circ}$ (FWHM) at a distance of $\sim 3^{\prime \prime}(4.5 \mathrm{kpc})$ beyond the second knot, $\mathrm{K}_{2}$.

6. The total-intensity morphology of the $\mathrm{S}$ lobe is somewhat peculiar at both 6 and $21 \mathrm{~cm}$. A photographic representation of the $21 \mathrm{~cm}$ map (Fig. 3, Pl. 10) suggests the presence of a band of diminished intensity (henceforth referred to as "band") across the center of the lobe, oriented perpendicular to the jet axis. This band is located where the widened jet fades out into the lobe, and where the percentage polarization exhibits a "trough" at $6 \mathrm{~cm}$ (see next section).

\section{ii) Polarization}

The polarization distribution of 3 C 277.3 is unusual, particularly in the southern half of the source:

1. The percentage polarization at $6 \mathrm{~cm}$ and $21 \mathrm{~cm}$ is generally low except in some places along the edges of the lobes (see Fig. 4, panels 5 and 6). Our $2 \mathrm{~cm}$ map is rather noisy, and significant polarization was detected only from the outermost extremity of the northern hot spot.

2. The most striking polarization feature is a long, straight, and narrow region of low polarization along the jet (seen best at $6 \mathrm{~cm}$ in Fig. 4, panel 5, but also discernible at $21 \mathrm{~cm}$ in panel $6)$. This feature connects the second knot of the southern jet $\left(\mathrm{K}_{2}\right)$ to the brightness enhancement and the polarization "trough" (see below) near the center of the $S$ lobe. The $6 \mathrm{~cm}$ percentage polarization is $16 \%$ in this feature and $26 \%$ on either side (east and west).

3. Another curious feature at $6 \mathrm{~cm}$ is a "trough" of low $(\lesssim 3 \%)$ polarization cutting across the middle of the $S$ lobe at a right angle to the jet axis (see Fig. 4, panel 5). The narrow 


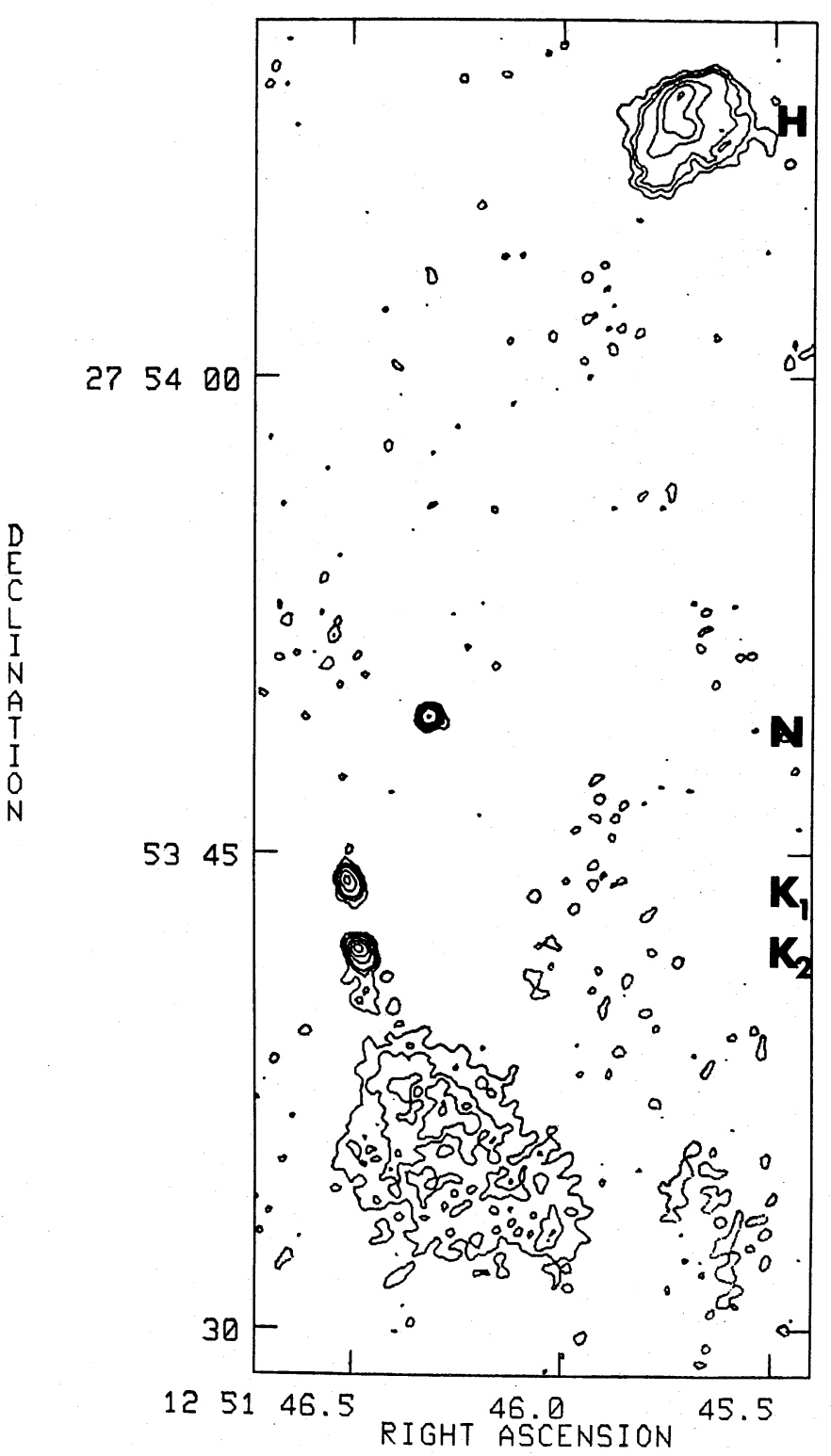

FIG. 2.-Contour representation of the total intensity of $3 \mathrm{C} 277.3$ at $6.2 \mathrm{~cm}$ $\left(0^{\prime \prime} 4 \times 0\right.$ " 4 resolution). Some features are labeled (as in Fig. 1). Contour values (in mJy per beam) are $0.12 \times(-2,2,4,6,12,20,30,90)$. Negative values would have appeared dashed.

jetlike region of low polarization discussed immediately above merges with this trough near the center of a brightness enhancement in the total intensity. The polarization position angle at $6 \mathrm{~cm}$ changes abruptly (by $\sim 90^{\circ}$ ) across this trough. At $21 \mathrm{~cm}$ (see Fig. 4, panel 6) the polarization drops abruptly (to $\lesssim 2 \%$ ) at the position of the trough but (unlike at $6 \mathrm{~cm}$ ) does not rise to the SW of the trough. The band of low brightness in the total intensity discussed above coincides with the central portion of this trough.

4. The northern hot spot and adjacent regions in the lobe are unpolarized at $6 \mathrm{~cm}(\lesssim 1.1 \%)$ except for a small region at the northernmost extremity $(\sim 5 \% \pm 1 \%)$ where $2 \mathrm{~cm}$ polarized flux was also detected $(39 \% \pm 6 \%)$. iii) Spectral Comparison

We have compared the 1 ". $2 \times 1$ ". 2 resolution maps of the total and polarized intensity at each wavelength to obtain the spectral index, depolarization, and differential polarization position angle distributions. The flux densities for the compact components $\left(\mathrm{N}, \mathrm{K}_{1}, \mathrm{~K}_{2}\right)$ were obtained using Gaussian model fits, and for the larger components, by integration over the appropriate areas in the 1.2 maps.

The following conventions are used: $(a)$ The spectral index $(\alpha)$ is defined by $S_{v} \sim v^{-\alpha}$. (b) The depolarization parameter (DP) is the ratio of the percentage polarization at $21 \mathrm{~cm}(6 \mathrm{~cm})$ to that at $6 \mathrm{~cm}(2 \mathrm{~cm}) .(c)$ The rotation $(\Delta \phi)$ of the polarization position angles is defined as the position angle at $21 \mathrm{~cm}(6 \mathrm{~cm})$ minus the angle at $6 \mathrm{~cm}(2 \mathrm{~cm})$.

The results are given in Tables 4 and 5. Profiles illustrating the variation of these parameters along the southern jet and central lobe are shown in Figure 5. We can summarize these results as follows:

1. The radio spectrum steepens between $21 \mathrm{~cm}$ and $2 \mathrm{~cm}$ in the hot spot $(\mathrm{H})$ and the knots $\left(\mathrm{K}_{1}\right.$ and $\left.\mathrm{K}_{2}\right)$.

2. The knot farther from the nucleus $\left(\mathrm{K}_{2}\right)$ is the brighter and has the flatter spectrum of the two knots. Note that the optical continuum is mostly associated with knot $\mathrm{K}_{1}$ (Miley et al. 1981).

3. Beyond knot $\mathrm{K}_{2}$, the spectral index $\left(\alpha_{6}^{21}\right)$, percentage polarization at $6 \mathrm{~cm}$, depolarization ratio $\left(\mathrm{DP}_{6}^{21}\right)$, and rotation $\left(\Delta \phi_{6}^{21}\right)$ all tend to increase along the jet with increasing distance from the core. This trend is not followed beyond the $6 \mathrm{~cm}$ polarization trough: in the soutwestern half of the lobe, typically $\mathrm{DP}_{6}^{21} \leq 0.15$.

4. Outside the jet, in the inner southern lobe north of the trough, the spectral index, depolarization ratio, and rotation do not vary significantly. Typically $\alpha_{6}^{21}=0.68 \pm 0.03, \mathrm{DP}_{6}^{21}=$ $0.95 \pm 0.10, \Delta \phi_{6}^{21}=5^{\circ} \pm 10^{\circ}$.

5. The spectral indices $\alpha_{6}^{20}$ in the middle of the southern lobe and in the northern lobe have values of $\sim 0.7$, similar to that in the jet.

6. At the northern extremity of the northern hot spot, $\mathrm{DP}_{6}^{20} \leq 0.17, \mathrm{DP}_{2}^{6}=0.13 \pm 0.03, \Delta \phi_{2}^{6}=22^{\circ} \pm 8^{\circ}$.

$$
\text { iv) Physical Parameters }
$$

We have used the VLA maps and the spectral index distribution to derive the various physical parameters listed in Table 4. The most important, ad hoc, assumptions we have used are (e.g., Pacholczyk 1970) (a) The total energy of a component is minimal as a function of magnetic field strength. (b) The relativistic electron and proton energies are equal. (c) The filling factor of the synchrotron emission is unity. (d) The nonthermal spectrum is a power law extending from $10 \mathrm{MHz}$ to $100 \mathrm{GHz}$. (e) The volumes of the individual components are assumed to be of cylindrical form (or spherical if the minor and major axes are comparable).

\section{b) Optical}

\section{i) Morphology: Comparison with the Radio Maps}

Various overlays of the radio and optical maps are shown in Figure 4 (panels 1, 2, 3, 4). To align the maps, it was assumed that the nucleus of the galaxy and the central compact radio component coincide. With this assumption we estimate that the relative alignments of the radio and optical features are accurate to less than $0^{\prime \prime} 2$.

In all four panels the gray scales represent the optical data. The faint, diffuse optical emission outside the galaxy is best 

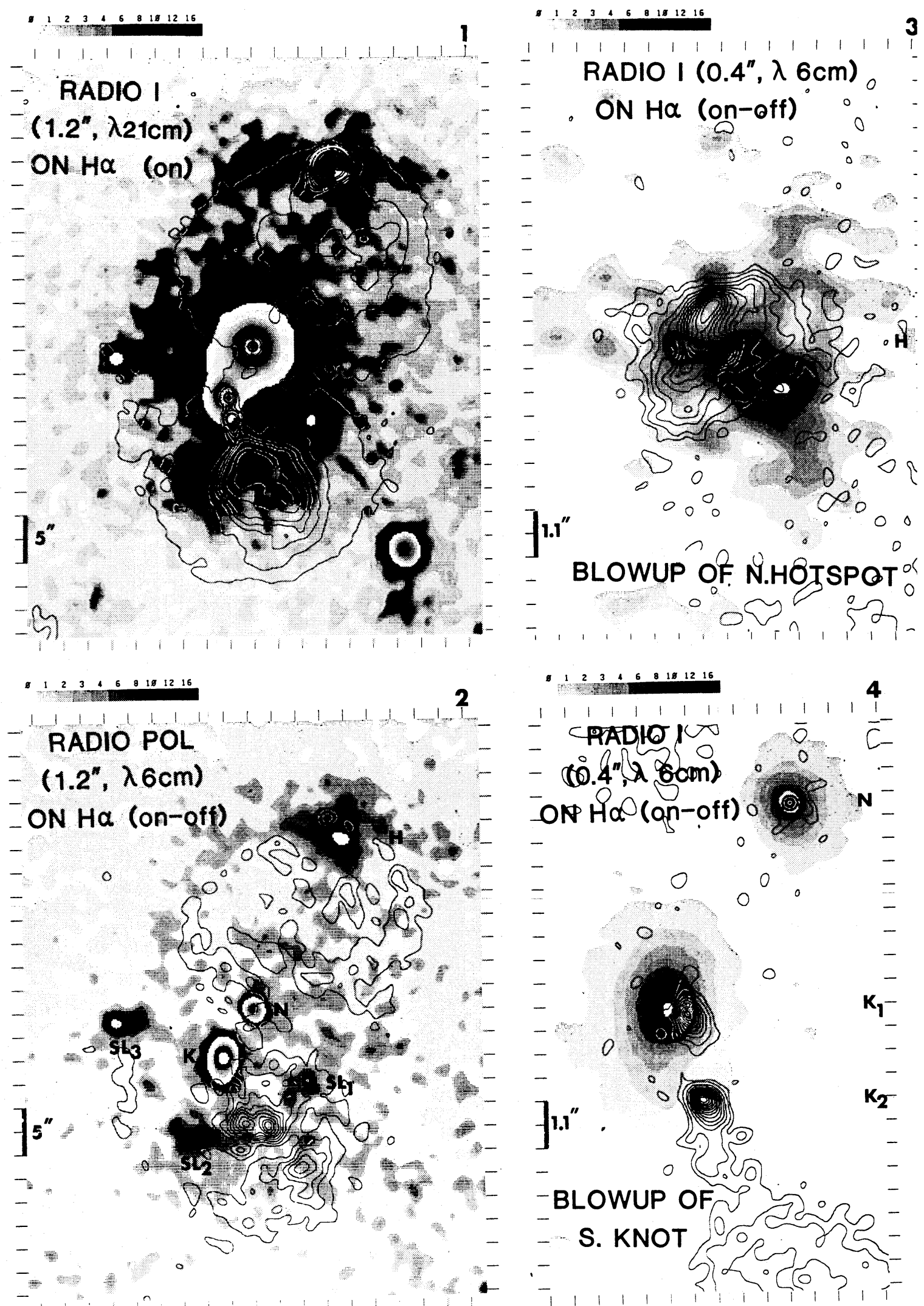

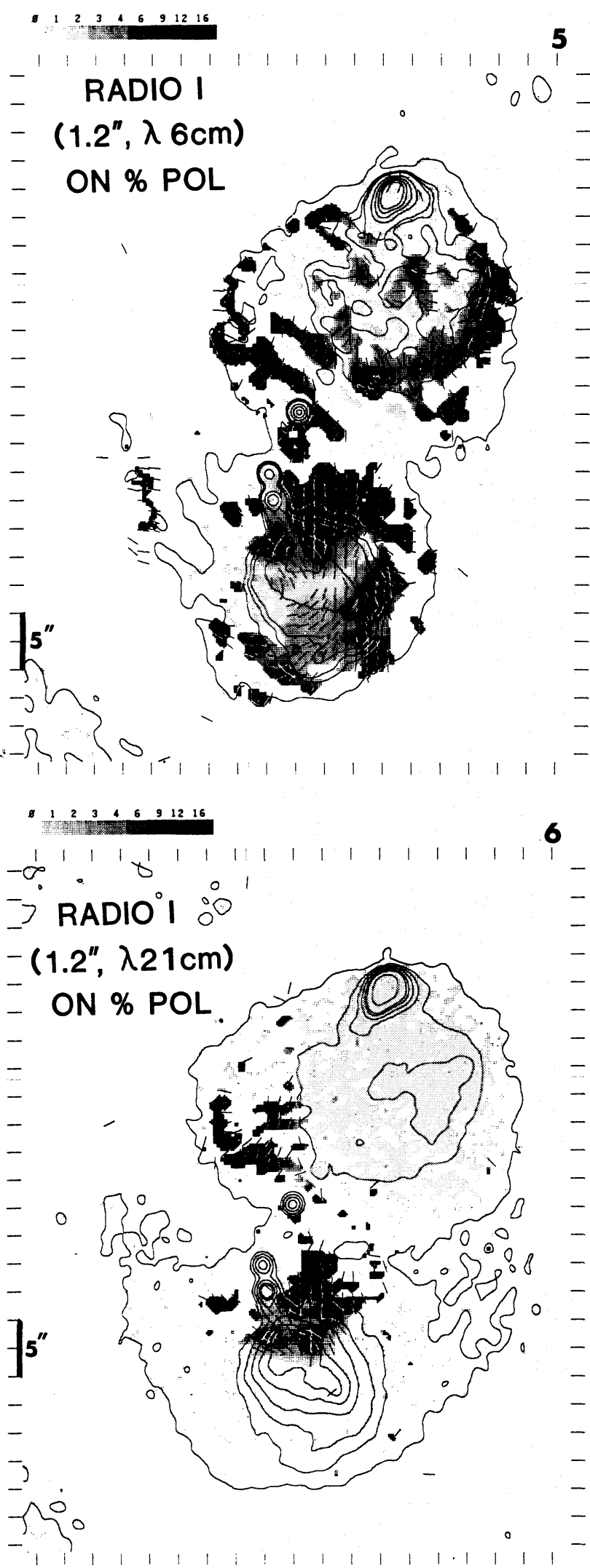

Fig. 4.-Various radio/optical overlays of $3 \mathrm{C} 277.3$ to illustrate the morphological relationships of the line-emitting gas and the radio continuum of this source. Some features are labeled for the reference in the text (see also Fig. 1). Panel 1, Gray scales represent the $\mathrm{H} \alpha+[\mathrm{N} \mathrm{II}]$ emission smoothed to $\sim 1.5$ resolution with no continuum subtracted. The contours represent the total intensity at $21 \mathrm{~cm}$ with $1^{\prime \prime} .2 \times 1^{\prime \prime} .2$ resolution. Contour values are $0.6,3,5,8,12$, $28 \mathrm{mJy}$ per beam. Panel 2, Gray scales represent the pure $\mathrm{H} \alpha+[\mathrm{N} \mathrm{II}]$ emission smoothed to $\sim 1$ 1" 5 resolution (continuum subtracted). The contours represent the polarized intensity at $6 \mathrm{~cm}$ with 1 ". $2 \times 1$ 1".2 resolution. Contour values are from 0.3 to 2.7 in steps of $0.3 \mathrm{mJy}$ per beam. Panel 3, Gray scales represent the pure $\mathrm{H} \alpha+[\mathrm{N}$ II] emission smoothed to $\sim 0$ " 8 resolution, near the northern hotspot $(\mathrm{H})$. The contours represent the total intensity at $6 \mathrm{~cm}$ with $0.4 \times 0.4$ resolution. Contour values are $0.15,0.3,0.6,1.2,1.5,1.8,2.1,2.4,2.7,3.0,3.3$, 3.6, 3.9, 4.2, 7.5, $11 \mathrm{mJy}$ per beam. Panel 4, As in panel 3 for the nucleus and the knots $\left(\mathrm{K}_{1}, \mathrm{~K}_{2}\right)$ in the jet. Panel 5 , Gray scales represent the percentage polarization at $6 \mathrm{~cm}$ with $1.2 \times 1.2$ resolution. Gray scale values ranging from 0 to 16 (see top of panel) correspond to percentage polarization as follows: $0,0 \% ; 1$, $0 \%-5 \% ; 2,5 \%-10 \% ; 3,10 \%-15 \% ; 4,15 \%-20 \% ; 6,20 \% ; 9,25 \%-30 \% ; 12$, $30 \%-50 \% ; 16,50 \%-90 \%$. The contours represent the total intensity at $6 \mathrm{~cm}$ with 1 ."2 2 1".2 resolution. Contour values are $0.5,2,2.5,3,6,9,12,15 \mathrm{mJy}$ per beam. The bars represent the polarization position angles; bar lengths are arbitrary. Panel 6 , As in panel 5 for $21 \mathrm{~cm}$. Contour values are $0.55,3,5,8,12$, $15,28 \mathrm{mJy}$ per beam. 


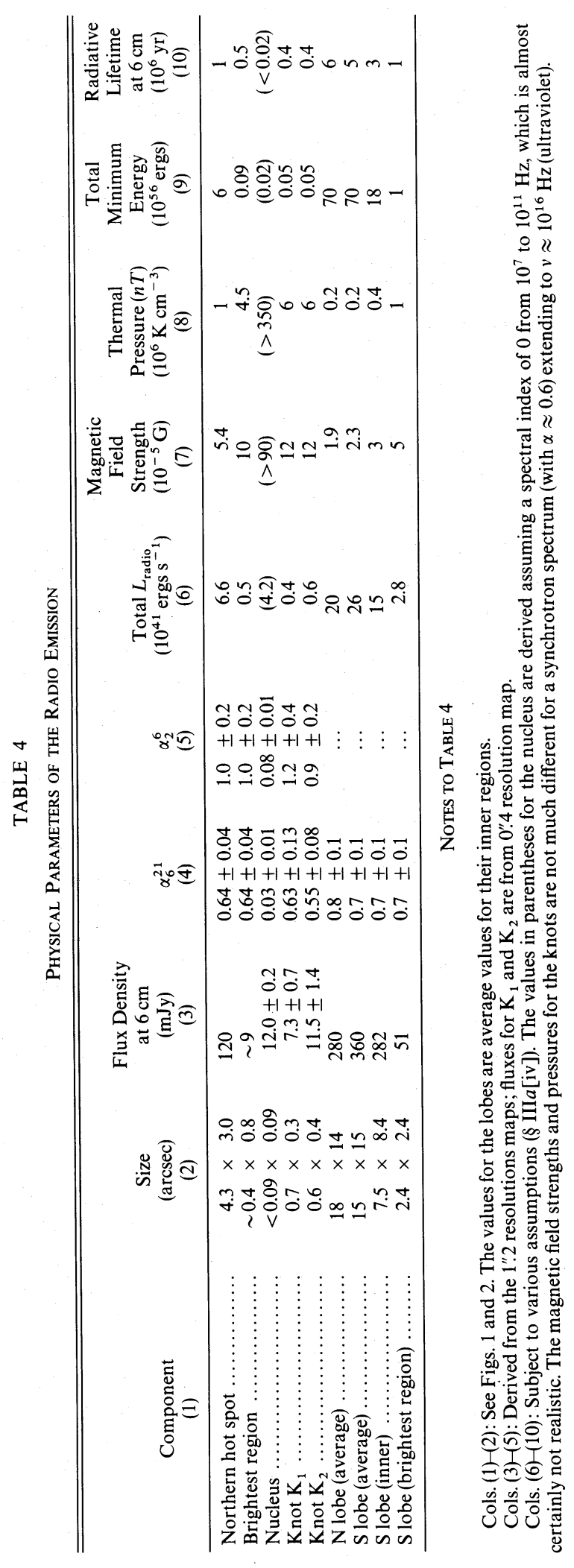

505 
TABLE 5

Positions of COMPACT COMPONENTS

\begin{tabular}{ccc}
\hline \hline \multicolumn{1}{c}{ Component } & R.A. (1950) & Decl. (1950) \\
\hline Nucleus $\ldots \ldots \ldots$ & $12^{\mathrm{h}} 51^{\mathrm{m}} 46^{\mathrm{s}} .316 \pm 0^{\mathrm{s}} 001$ & $+27^{\circ} 53^{\prime} 49^{\prime \prime} .34 \pm 0^{\prime \prime} 01$ \\
Knot $\mathrm{K}_{1} \ldots \ldots \ldots$ & $125146.506 \pm 0.001$ & $+275344.06 \pm 0.01$ \\
Knot $\mathrm{K}_{2} \ldots \ldots$ & $125146.480 \pm 0.001$ & $+275341.90 \pm 0.01$ \\
\hline
\end{tabular}

shown in panel 1 overlaid with the $21 \mathrm{~cm}$ map. In this panel the optical continuum was not subtracted, to avoid signal-to-noise degradation. The pure $\mathrm{H} \alpha+[\mathrm{N}$ II $]$ line emission (continuum from galaxy and knot subtracted) is shown in panel 2 overlaid with the $6 \mathrm{~cm}$ polarization map and in panels 3 and 4 overlaid with the high-resolution $6 \mathrm{~cm}$ total-intensity maps. The original, unsmoothed data are also photographically reproduced in Figures $6 a$ and $6 b$ (Plates 11 and 12).

We conclude from these figures:

1. The emission-line gas is extended by $\sim 65 \mathrm{kpc} \mathrm{N-S}$ and $\sim 40 \mathrm{kpc} \mathrm{E}-\mathrm{W}$. The northernmost gas is located at a projected distance of $\sim 40 \mathrm{kpc}$ from the nucleus. These dimensions are similar to those of the radio source.

2. The two brightest emission-line regions $(\mathrm{H}$ and $\mathrm{K})$ are found along the principal radio axis (as defined by the radio hot spot-the nucleus-jet knot $\mathrm{K}_{1}$ ). The bright southern feature $\mathrm{SL}_{2}$ is also located along this axis.

3. The emission-line gas is located primarily along the boundaries of the radio source, with the brightest regions $(H$ and $\mathrm{K}_{1}$ ) occurring adjacent to bright radio features (the northern hot spot and southern jet knot $\mathrm{K}_{1}$ ). This is well illustrated by Figure 4, panels 3 and 4 , which show that $H$ and $K_{1}$ are significantly offset from the corresponding radio knots. A particularly striking feature visible in Figure 4, panels 1 and 2, and Figure 6 is a faint loop of line emission extending along the NE boundary of the $\mathrm{N}$ lobe. Even emission-line region $\mathrm{SL}_{3}$, which consists of two compact components, is situated near the end of a low-brightness tail of radio emission (cf. Fig. 4, panels 6 and 2).
4. The presence of emission-line gas is anticorrelated with the percentage polarization at $6 \mathrm{~cm}$ (see Fig. 4, panel 2, and cf. panel 5). The two emission-line features $\mathrm{SL}_{1}$ and $\mathrm{SL}_{2}$ lie approximately at the extremities of the trough of low polarization in the $\mathrm{S}$ lobe (see above).

5. Our $\mathrm{H} \alpha+[\mathrm{N} \mathrm{II}]$ image (Fig. 4, panels 1 and 2) suggests that weak, patchy line emission occurs throughout the source in projection. The long-slit spectroscopic data confirm this.

\section{ii) Kinematics}

Velocity gradients.-In Figure 7 we plot the gas velocity along several position angles covered by our long-slit spectra: P.A. $=155^{\circ}$ through the nucleus of the galaxy, P.A. $=155^{\circ}$ through a point $10^{\prime \prime}$ due west of the nucleus, P.A. $=69^{\circ}$ passing through the jet knot $\mathrm{K}_{1}$ and the eastern feature $\mathrm{SL}_{3}$, and P.A. $=77^{\circ}$ along the boundary of the northern radio lobe (see Fig. 6). From these data we can conclude the following:

1. Strong velocity changes of $\sim 500-600 \mathrm{~km} \mathrm{~s}^{-1}$ are seen along or parallel to the radio axis (P.A. $=155^{\circ}$ ). Velocities increase to the north.

2. Small velocity changes of $\sim 50-100 \mathrm{~km} \mathrm{~s}^{-1}$ are seen perpendicular to the radio axis (P.A. $=69^{\circ}$ or $77^{\circ}$ ). Velocities increase to the east.

3. A particularly strong velocity gradient is seen just north of the northern radio spot (velocities increase by $\sim 200 \mathrm{~km} \mathrm{~s}^{-1}$ over 7", $10 \mathrm{kpc}$ ).

4. The velocity field along the radio axis is asymmetric with respect to the nucleus. Velocities are only $-25 \mathrm{~km} \mathrm{~s}^{-1}$ at $17^{\prime \prime} \mathrm{S}$ of the nucleus compared with $+250 \mathrm{~km} \mathrm{~s}^{-1}$ at $20^{\prime \prime} \mathrm{N}$ and $+400 \mathrm{~km} \mathrm{~s}^{-1}$ at $27^{\prime \prime} \mathrm{N}$.

Line widths.-The emission-line widths (the [O III] $\lambda 5007$ FWHM corrected for instrumental broadening) at various locations covered by our long-slit data are summarized in Table 6. Our principal conclusions from these data are:

1. The extranuclear line widths range from $\leq 100 \mathrm{~km} \mathrm{~s}^{-1}$ to $240 \mathrm{~km} \mathrm{~s}^{-1}$. These lines are somewhat narrower than the emission lines associated with the radio lobes in 3C 305, 3C 293, 4C

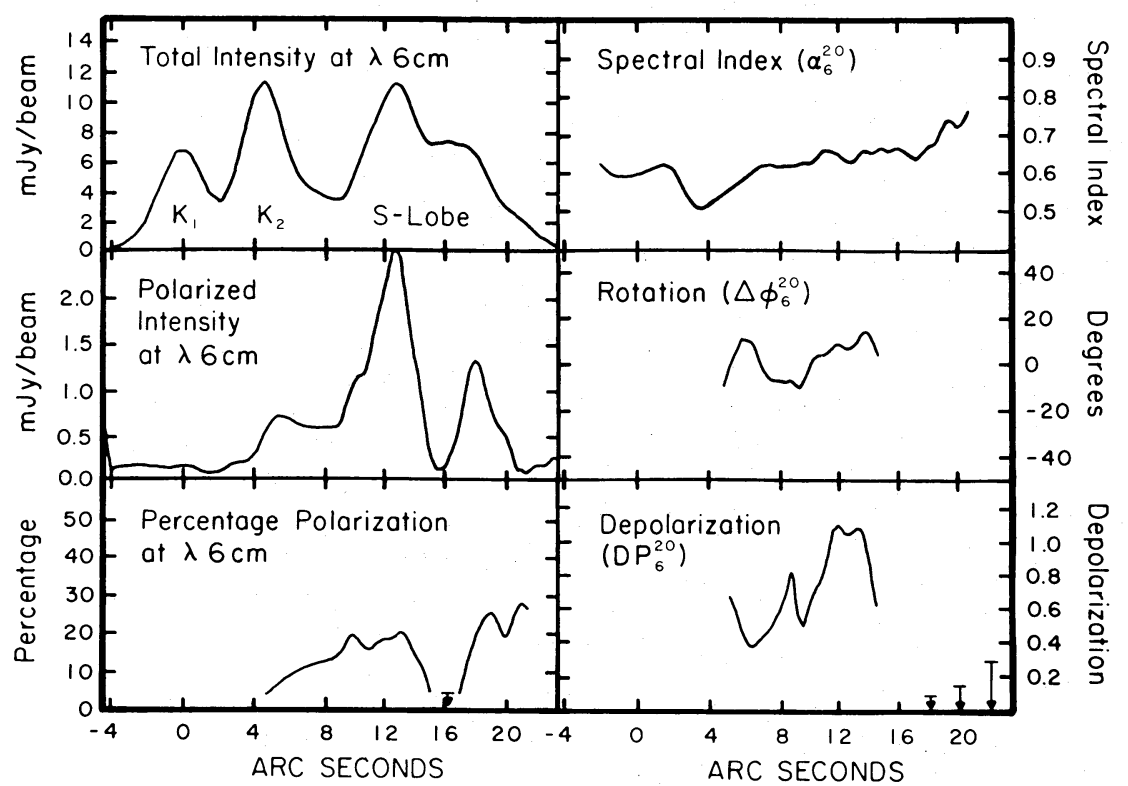

FIG. 5.-Profiles (1".2 resolution) through knots $\mathrm{K}_{1}, \mathrm{~K}_{2}$, and the southern lobe along the jet direction showing the variation in the total and polarized intensities and their spectral properties. 


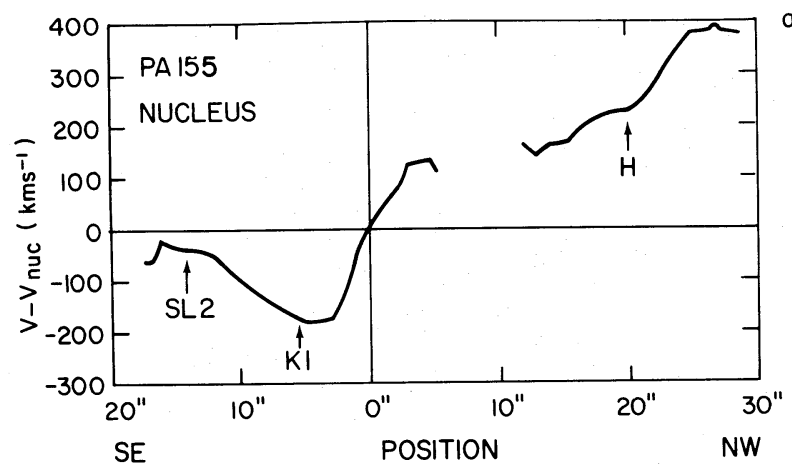

FIG. 7. (a) Velocity relative to the nucleus $\left(V=25,610 \mathrm{~km} \mathrm{~s}^{-1}\right)$ along P.A. $=155^{\circ}$ through the nucleus. The velocities were derived from the $[\mathrm{O}$ III $]$ $\lambda 5007$ line in our Cryogenic Camera data. The reference position is the nucleus. Uncertainties are $\$ 50 \mathrm{~km} \mathrm{~s}^{-1}$. (b) Velocity relative to nucleus in P.A. $155^{\circ}$ passing $10^{\prime \prime}$ west of the nucleus. Velocities were derived from Cryogenic Camera data using the $\mathrm{H} \alpha$ emission line. The reference position is $10^{\prime \prime}$ west of the nucleus. Uncertainties are $\sim 200 \mathrm{~km} \mathrm{~s}^{-1}$. (c) Velocity relative to the nucleus along P.A. $=69^{\circ}$ south of the nucleus, passing through jet knot $\mathrm{K}_{1}$ and the eastern feature $\mathrm{SL}_{3}$ (see text). The velocities were derived from HGVS data using the $[\mathrm{O} \mathrm{III}] 25007$ line. The reference position is $\mathrm{K}_{1}$ (jet). Uncertainties are $\leqslant 30 \mathrm{~km} \mathrm{~s}^{-1}$. (d) Velocities relative to the nucleus along P.A. $=77^{\circ}$ north of the nucleus, passing through the northern hot spot $(\mathrm{H})$. Velocities were derived from HGVS data using the $[\mathrm{O}$ III] $\lambda 5007$ line. Positions are given relative to a reference position $17^{\prime \prime} .6 \mathrm{~N}$ and $9^{\prime \prime} .2 \mathrm{~W}$ of the nucleus (the peak in $\mathrm{H} \alpha$ surface brightness in the $\mathrm{N}$ lobe). Uncertainties are $\sim 30 \mathrm{~km} \mathrm{~s}^{-1}$.

26.42, 4C 29.30, and 3C 171 (Heckman et al. 1982; van Breugel et al. 1984; van Breugel, Heckman, and Miley 1984, 1985; Heckman, van Breugel, and Miley 1984).

2. The extranuclear lines are broader in the regions adjacent to strong radio emission (i.e., $240 \mathrm{~km} \mathrm{~s}^{-1} 2^{\prime \prime}$ from the northern hot spot vs. $170 \mathrm{~km} \mathrm{~s}^{-1} 8^{\prime \prime}$ from the northern hot spot; $170 \mathrm{~km}$ $\mathrm{s}^{-1}$ near the jet $\mathrm{K}$, vs. less than $100 \mathrm{~km} \mathrm{~s}^{-1}$ in the eastern feature $\mathrm{SL}_{3}$ ). Such a trend has also been reported for some of the other sources mentioned above.

iii) Emission-Line Spectrum

Table 7 summarizes the relative intensities of the emission lines detected at various locations in $3 \mathrm{C}$ 277.3. The emissionline spectra (uncalibrated in flux) are shown for several representative locations in Figure 8 . From our data we can conclude the following:

1. In general the spectrum of the gas near the jet $\left(\mathrm{K}_{1}\right)$ resem-
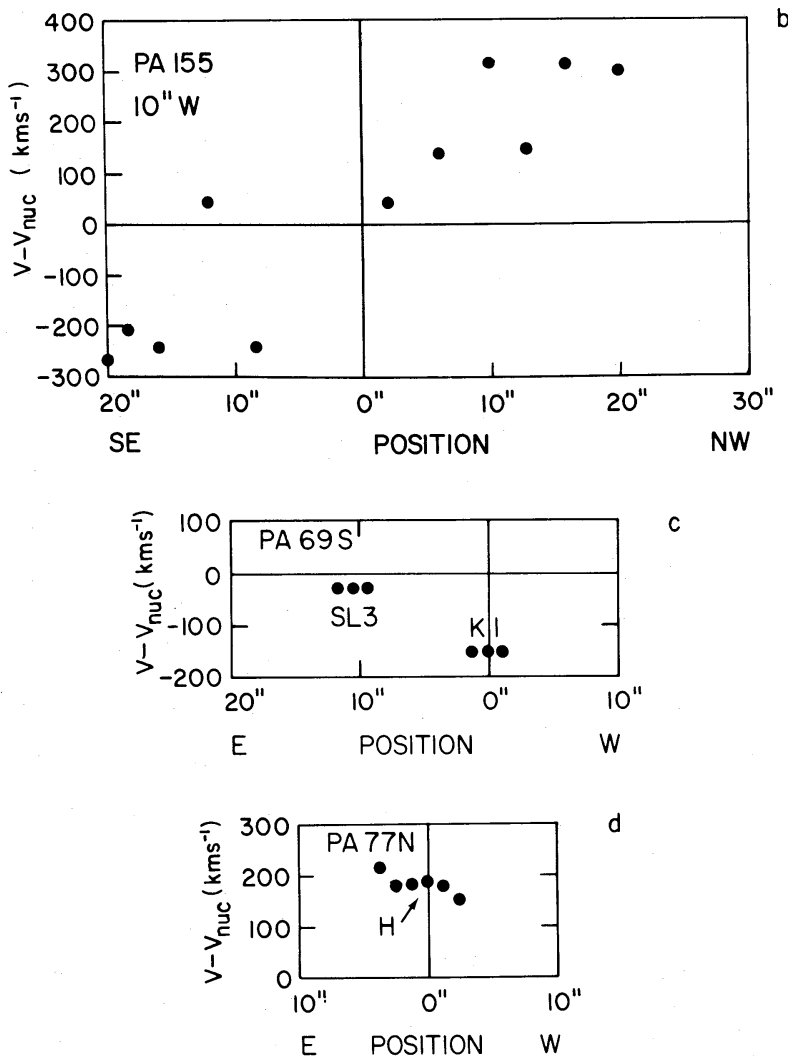

bles the spectrum of a type 2 Seyfert galaxy (Koski 1978). The spectrum of the gas near the $\mathrm{N}$ hot spot $(\mathrm{H})$ and in the nucleus $(\mathrm{N})$ is of lower excitation and appears intermediate in character

TABLE 6

EMISSION-LINE WIDTHS

\begin{tabular}{cc}
\hline \hline Position & {$[\mathrm{O}$ III] $25007 \mathrm{FWHM}$} \\
\hline Nucleus $\ldots \ldots \ldots \ldots \ldots \ldots$ & $460 \mathrm{~km} \mathrm{~s}^{-1}$ \\
N hot spot: & \\
$2^{\prime \prime} \mathrm{N}$ of hot spot $\ldots \ldots \ldots$ & $240 \mathrm{~km} \mathrm{~s}^{-1}$ \\
$8^{\prime \prime} \mathrm{N}$ of hot spot $\ldots \ldots \ldots$ & $170 \mathrm{~km} \mathrm{~s}^{-1}$ \\
Jet $(\mathrm{K}) \ldots \ldots \ldots \ldots \ldots \ldots$ & $170 \mathrm{~km} \mathrm{~s}^{-1}$ \\
\hline
\end{tabular}

TABLE 7

Relative EMission-Line INTENSITIES

\begin{tabular}{|c|c|c|c|c|c|}
\hline Line & Jet & Nucleus $^{\mathrm{a}}$ & N Lobe ${ }^{a}$ & Seyfert ${ }^{\mathrm{b}}$ & Linear $^{c}$ \\
\hline$[\mathrm{S} \mathrm{II}] \lambda \lambda 6717+6731 \ldots \ldots$. & 15.5 & 30 & 12 & 16 & 30 \\
\hline$[\mathrm{N}$ II $] \lambda 6584 \ldots \ldots \ldots \ldots \ldots$ & 6.5 & 45 & 4.5 & 44 & 30 \\
\hline $\mathrm{H} \alpha \ldots \ldots \ldots$ & 40 & $\equiv 30$ & $\equiv 30$ & 29 & 29 \\
\hline$\left[\mathrm{O}_{1}\right] \lambda 6300 \ldots$ & 5.5 & 45 & 4.5 & 4 & 15 \\
\hline$[\mathrm{O} \mathrm{III}] \lambda 5007$. & 104 & 100 & 45 & 110 & 22 \\
\hline $\mathrm{H} \beta \ldots \ldots \ldots \ldots$ & $\equiv 10$ & $\equiv 10$ & $\equiv 10$ & $\equiv 10$ & $\equiv 10$ \\
\hline$[\mathrm{O}$ III] $\lambda 4363$. & $<2.3$ & $<10$ & $<7$ & 2 & 1.8 \\
\hline $\mathrm{H} \gamma \quad \ldots \ldots \ldots \ldots$ & 4.9 & $<10$ & $<7$ & 2 & 4.8 \\
\hline$[\mathrm{Ne} \mathrm{III}] \lambda 3869 \ldots \ldots \ldots \ldots$ & 5.4 & $<10$ & $\lesssim 15$ & 11 & 6 \\
\hline$\left[\begin{array}{lll}\mathrm{O} & \mathrm{II}\end{array}\right] \bar{\lambda} 3727 \ldots \ldots \ldots$ & 25 & 100 & 80 & 35 & 80 \\
\hline
\end{tabular}

The relative intensities of the lines in the red $\left(\left[\mathrm{O}_{\mathrm{I}}\right]-[\mathrm{S} \mathrm{II}]\right)$ cannot be reliably ascertained with respect to the lines in blue-green $([\mathrm{O} \mathrm{II}]-[\mathrm{O} \mathrm{III}])$ for the nucleus and the $\mathrm{N}$ lobe (see $\S \mathrm{II})$. We have normalized these two portions of the spectra by taking $\mathrm{H} \beta \equiv 10$ and $\mathrm{H} \alpha \equiv 30$.

b From Koski 1978.

${ }^{\mathrm{c}}$ From Fosbury et al. 1978 


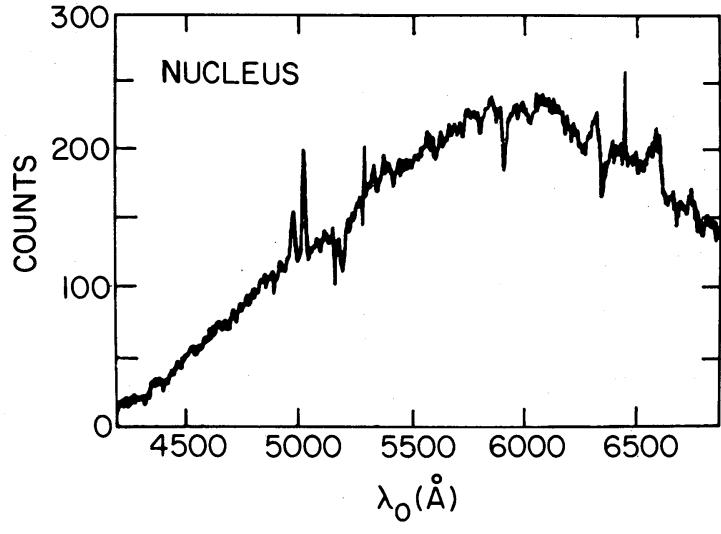

FIG. $8 a$

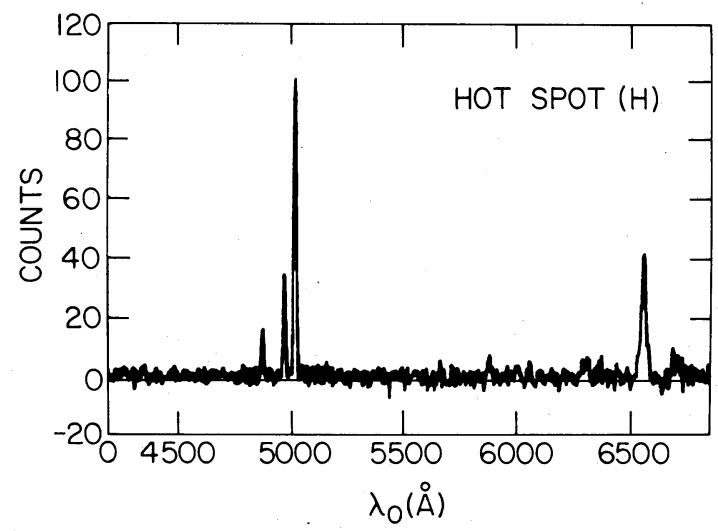

Fig. $8 c$

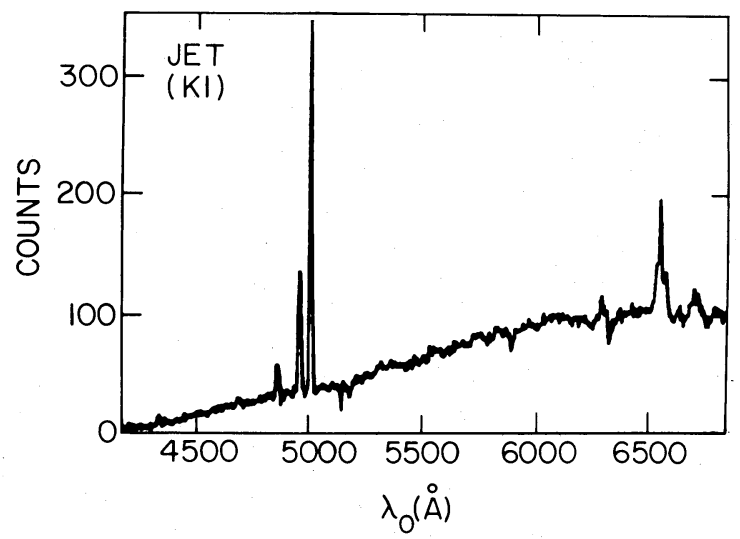

FIG. $8 b$

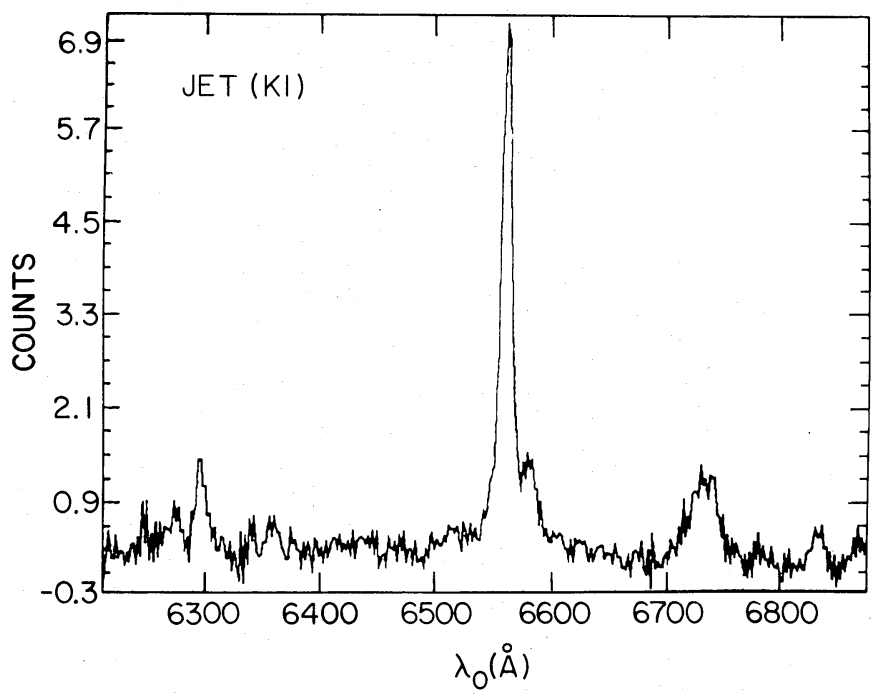

FIG. $8 d$

Fig. 8. (a) Cryogenic Camera spectrum of the nucleus of $3 \mathrm{C} 277.3$. The wavelength scale is in the frame of the emitting material, and the intensity is in arbitrary units (not calibrated in either relative or absolute flux). (b) Spectrum of the jet knot $\mathrm{K}_{1}$ (see text). All else as in Fig. 8a. (c) Spectrum of the $\mathrm{N}$ radio hot spot (see text). All else as in Fig. 8a. (d) Red Reticon spectrum of the jet knot $\mathrm{K}_{1}$ (see text). All else as in Fig. $8 a$.

between a Seyfert 2 and a Liner (low-ionization nuclear emission-line region) (Heckman 1980b; Baldwin, Phillips, and Terlevich 1981).

2. The most peculiar feature of the spectra of the gas near the jet $\left(\mathrm{K}_{1}\right)$ and hot spot $(\mathrm{H})$ is the relative weakness of [N II] $\lambda 6584$ compared with its strength in the spectra of the nucleus of 3C 277.3 or of typical Liners and Seyfert galaxies. Rubin, Ford, and Whitmore (1984) show that the ratio of the [N II] $\lambda \lambda 6584,6548$ to [S II] $\lambda \lambda 6717,6731$ lines in $\mathrm{H}$ II regions is a strong function of galaxy absolute magnitude (since the $\mathrm{N} / \mathrm{S}$ abundance increases as a function of overall metal abundance, which in turn increases with galaxy luminosity). In 3C 277.3, the $\log ([\mathrm{N} \mathrm{II}] /[\mathrm{S} \mathrm{II}])$ value in the nucleus $(+0.30)$ is consistent with that expected in a galaxy of $M_{v} \sim-20$ to -22 (Rubin et al.). However, in the jet and $\mathrm{N}$ lobe the $\log$ ratios $(-0.25$ and $-0.30)$ are similar to values found in low-luminosity $\left(M_{v} \geq\right.$ -18), metal-poor galaxies. The power-law photoionization models of Ferland and Netzer (1983) and the shock models of Dopita (1977) and Raymond (1979) also all suggest subsolar metal abundances for the extranuclear gas in 3C 277.3 (e.g., Ferland and Netzer's models imply $\mathrm{O} / \mathrm{H}$ down by a factor of $\sim 3$ from solar, but $\mathrm{N} / \mathrm{H}$ down by a factor $\sim 10$ ). In particular, photoionization models of gas with abundances similar to those in $\mathrm{H}$ il regions in the Magellanic Clouds yield good matches to our observed spectra (Kramer 1984). This is a potentially important clue to the origin of this gas.

3. The nature of the emission-line spectrum of $\mathrm{SL}_{3}$ is unclear. We know only that [O III] $\lambda 5007 / \mathrm{H} \beta \sim 3$, consistent with an H II region or Liner (Heckman $1980 b$; Baldwin, Phillips, and Terlevich 1981).

4. Reddening in knot $\mathrm{K}_{1}$ is modest. Assuming a normal case $B$ value for the intrinsic $\mathrm{H} \alpha / \mathrm{H} \beta$ ratio implies $\sim 1.5 \mathrm{mag}$ of extinction at $\mathrm{H} \beta$.

5. The density-sensitive ratio of $[\mathrm{S}$ II] $\lambda 6717$ to $[\mathrm{S}$ II $] \lambda 6731$ is $\sim 1.15 \pm 0.2$ in knot $\mathrm{K}_{1}$, implying an electron density of $n_{e} \sim 600 \mathrm{~cm}^{-3}$ (to within a factor $\sim 2$ ). (However, see note added in proof). It was not possible to estimate $n_{e}$ elsewhere since the $[\mathrm{S} \mathrm{II}]$ lines were too faint to measure accurately.

6 . The limit to the intensity ratio [O $\left[\begin{array}{ll}\mathrm{O} & \mathrm{III}\end{array} \lambda 4363\right.$ to [O III] $\lambda 5007$ in knot $\mathrm{K}_{1}$ implies $T_{e} \lesssim 16,000 \mathrm{~K}$, consistent with photoionization but inconsistent with collisional heating by slow $\left(V \lesssim 200 \mathrm{~km} \mathrm{~s}^{-1}\right.$ ) shock waves (see below). Near the hot 
TABLE 8

Physical Parameters of the Line-emitting Gas

\begin{tabular}{|c|c|c|c|c|c|c|}
\hline $\begin{array}{c}\text { Component } \\
\text { (1) }\end{array}$ & $\begin{array}{c}\text { Area } \\
(\operatorname{arcsec}) \\
(2)\end{array}$ & $\begin{array}{c}\text { Integrated } \\
\text { Flux } \mathrm{H} \alpha+[\mathrm{N} \mathrm{II}] \\
\left(10^{-15} \mathrm{ergs}^{\left.-\mathrm{cm}^{-2} \mathrm{~s}^{-1}\right)}\right. \\
\text { (3) }\end{array}$ & $\begin{array}{c}\mathrm{H} \alpha+[\mathrm{N} \mathrm{II}] \\
\text { Luminosity } \\
\left(10^{40} \mathrm{erg}^{-1}\right) \\
(4)\end{array}$ & $\begin{array}{c}\text { Filling } \\
\text { Factor } \\
{\left[10^{-8}(n / 600)^{-2}\right]} \\
(5)\end{array}$ & $\begin{array}{c}\text { Mass of Ionized } \\
\text { Hydrogen } \\
{\left[10^{5}(n / 600)^{-1} M_{\odot}\right]} \\
(6)\end{array}$ & $\begin{array}{c}\text { Kinetic } \\
\text { Energy } \\
{\left[10^{52}(n / 600)^{-1}(V / 150)^{2} \text { ergs }\right]} \\
(7)\end{array}$ \\
\hline Hot spot $\ldots \ldots \ldots \ldots \ldots$ & $8.5 \times 9.2$ & $4.1(0.3)$ & $5.7(0.4)$ & $1 \quad(80)$ & $2.8(0.2)$ & $6.5(0.5)$ \\
\hline Nucleus $\ldots . . . . . \ldots \ldots$ & $3.2 \times 3.2$ & $3.4(1.5)$ & $4.7(2.1)$ & $20(400)$ & $2.3(1.0)$ & $\ldots$ \\
\hline $\operatorname{Knot}\left(\mathrm{K}_{1}+\mathrm{K}_{2}\right) \ldots \ldots$ & $4.6 \times 6.4$ & $28 \quad(6.0)$ & $35 \quad(8.3)$ & $40 \quad(1700)$ & $17 \quad(4.0)$ & $39 \quad(8)$ \\
\hline S lobe $1 \ldots \ldots \ldots \ldots$ & $2.8 \times 4.2$ & $1.2(0.3)$ & $1.7(0.4)$ & $8 \quad(80)$ & $0.8(0.2)$ & $2.0(0.5)$ \\
\hline S lobe $2 \ldots \ldots \ldots \ldots$ & $2.8 \times 4.2$ & $0.9(0.1)$ & $1.3(0.1)$ & $6 \quad(30)$ & $0.7(0.05)$ & $1.5(0.1)$ \\
\hline $\mathrm{S}$ lobe $3 \ldots \ldots \ldots \ldots$ & $3.5 \times 5.7$ & $1.5(0.4)$ & $2.1(0.6)$ & $5 \quad(170)$ & $1.0(0.3)$ & $2.3(0.7)$ \\
\hline Total $\ldots \ldots \ldots \ldots \ldots$ & $33 \times 46$ & 39 & 50 & 0.1 & 23 & 52 \\
\hline
\end{tabular}

Cols. (1)-(2): See Fig. 4.

NOTES TO TABLE 8

Col. (3): Uncertainties are $\lesssim 20 \%$.

Col. (4): The total emission-line luminosity, assuming case B recombination (Osterbrock 1974), is approximately 20 times this value. Only the H $\alpha$ luminosity in the jet has been corrected for extinction.

Cols. (5)-(7): Assuming case B recombination. Volumes were calculated assuming cylindrical symmetry for each component.

Cols. (3)-(7): The values in parentheses are derived for small regions $\left(1^{\prime \prime} 1 \times 1^{\prime \prime} .1\right)$ centered at the peaks of the optical line emission.

spot (and elsewhere) the implied upper limit to $T_{e}$ is too large to be useful in this sense.

iv) Nuclear Optical Continuum

The equivalent widths of the $\lambda 5174 \mathrm{Mg}$ I and $\lambda 5892 \mathrm{Na}$ I stellar absorption lines are $\sim 6.5 \AA$ and $5 \AA$, respectively, in the nucleus of the parent galaxy of 3C 277.3. Since these values are quite normal for early-type galaxies (e.g., Heckman 1980a), we conclude that no strong nonstellar component is present in the nuclear spectrum. Yee and Oke (1978) found that a featureless continuum contributed only $3 \%-6 \%$ to the nuclear spectrum, consistent with the above.

\section{v) Physical Parameters of the Gas}

Various physical parameters of the line-emitting gas are given in Tables 8 and 9. The filling factor, mass of ionized hydrogen, and the kinetic energy were derived assuming a case $\mathrm{B}$ recombination spectrum and $T=1.5 \times 10^{4} \mathrm{~K}$ (Osterbrock 1974). In the jet $\left(K_{1}\right)$, we have corrected the $H \alpha$ luminosity for the measured extinction. Elsewhere we have assumed negligible extinction.

The parameters for each component were evaluated for two different regions: a large region which encompassed all the visible optical emission and a $11^{\prime \prime} 1 \times 1$." 1 region centered at the peaks of the optical emission. The filling factors were derived by assuming cylindrical symmetry for the volume within which the line-emitting gas is found. Because the gas is probably distributed in shells surrounding the radio source, the assumption of cylindrical symmetry would underestimate the filling factor of the gas in such shells.

The thermal electron density $\left(n_{e}\right)$ is measured only near the jet: $n_{e}=600 \mathrm{~cm}^{-3}$ within a factor of 2 . We have normalized all the derived parameters in columns (5), (6), and (7) of Table 8 with respect to this value.

Assuming that $T_{e} \sim 1.5 \times 10^{4} \mathrm{~K}$ in the gas near the jet (consistent with our limit, and typical of $T_{e}$ in Seyfert 2 galaxies according to Koski 1978), the thermal pressure in the emissionline gas is $2 n_{e} T \approx 2 \times 10^{7} \mathrm{~K} \mathrm{~cm}^{-3}$. This is similar to the minimum-energy value for the pressure in the radio plasma in the knots (Table 4). Thus, the line-emitting gas in the jet could be in rough pressure equilibrium with the radio plasma. A similar result has been found for the small radio source 3C 305 (Heckman et al. 1982).

If we assume that pressure equilibrium also holds in the other radio source components, then we can derive the thermal electron densities (for $T \approx 1.5 \times 10^{4} \mathrm{~K}$ ) and hence several other physical parameters of the optically emitting gas at these locations. These values are given in Table 9 and are highly model-dependent. The total mass of observed ionized hydro-

TABLE 9

Physical Parameters of the Line-emitting Gas Assuming Pressure Equilibrium WITH THE RADIO SOURCE

\begin{tabular}{|c|c|c|c|c|c|}
\hline $\begin{array}{l}\text { Component } \\
\text { (1) }\end{array}$ & $\begin{array}{c}\text { Radio } \\
\text { Pressure }(n T) \\
\left(10^{6} \mathrm{~K} \mathrm{~cm}^{-3}\right) \\
(2)\end{array}$ & $\begin{array}{c}n_{e}\left(\mathrm{~cm}^{-3}\right) \\
(3)\end{array}$ & $\begin{array}{l}\text { Filling } \\
\text { Factor } \\
\left(10^{-6}\right) \\
(4)\end{array}$ & $\begin{array}{c}M_{\mathrm{H}}^{+} \\
\left(10^{6} M_{\odot}\right) \\
\quad(5)\end{array}$ & $\begin{array}{c}\text { Kinetic } \\
\text { Energy } \\
{\left[10^{53}(\mathrm{~V} / 150)^{2} \mathrm{ergs}\right]} \\
(6)\end{array}$ \\
\hline $\mathrm{H}, \ldots \ldots \ldots \ldots \ldots$ & 1 & 33 & $4 \quad(120)$ & $5 \quad(0.4)$ & $12(1)$ \\
\hline $\mathrm{N}, \ldots \ldots \ldots \ldots \ldots$ & $>350$ & $>1170$ & $<0.05(0.6)$ & $<0.1(0.06)$ & $\ldots$ \\
\hline $\mathrm{K}_{1}+\mathrm{K}_{2} \ldots \ldots \ldots$ & $\sim 10$ & 330 & $0.8 \quad(27)$ & $3(0.8)$ & $7(2)$ \\
\hline $\mathrm{SL}_{1} \ldots \ldots \ldots \ldots \ldots$ & 0.2 & 7 & $400 \quad(2900)$ & $8 \quad(2)$ & $17(4)$ \\
\hline $\mathrm{SL}_{2} \ldots \ldots \ldots \ldots$ & 0.2 & $7 i$ & $(710)$ & $6 \quad(0.5)$ & $13(1)$ \\
\hline $\mathrm{SL}_{3} \ldots \ldots \ldots \ldots \ldots$ & 0.2 & 7 & $(4600)$ & $9 \quad(3)$ & $20(7)$ \\
\hline Total ........... & $\ldots$ & $\ldots$ & $\ldots$ & 31 & 70 \\
\hline
\end{tabular}

NoTES.-Values in parentheses in cols. (4)-(6) refer to the $1^{\prime \prime} 1 \times 1^{\prime \prime} .1$ region of peak brightness (see Table 8). 
gen in $3 \mathrm{C} 277.3$ would then be $\sim 3 \times 10^{7} M_{\odot}\left(\sim 2 \times 10^{6} M_{\odot}\right.$ near knot $\mathrm{K}_{1}$ is directly measured since we know $n_{e}$ there).

\section{DISCUSSION}

\section{a) Introduction}

The observational results summarized above clearly demonstrate that the nonthermal radio-emitting plasma and the thermal optically emitting gas in 3C 277.3 are closely related. We will therefore organize our discussion primarily in terms of this interaction. First, we will discuss the evidence that the line-emitting gas has a local origin (i.e., has not been transported out of the nucleus with the radio plasma). Once this is established, we will discuss how such local thermal material could affect the outbound radio plasma. When mixed into the boundary layers of the radio source, such material could depolarize the radio emission, while the collision between a radio jet and a massive gas cloud could can naturally account for many properties of the southern jet. The radio plasma in turn compresses, ionizes, and accelerates (entrains) the ambient gas. A continuous supply of entrained gas to the southern lobe by the jet could explain the large depolarization in this region. Finally, we will consider a model for the radio/optical emission in the northern radio hot spot. We show that the proximity of the two media, together with the limits on the X-ray luminosity of $3 \mathrm{C} 277.3$, seems to demand the presence of an inhomogeneous (cloudy) ambient medium in the halo of $3 \mathrm{C} 277.3$.

\section{b) Origin of the Gas}

It is of fundamental importance to know the origin of the line-emitting gas; in particular: (a) Is it gas moving with the jet-fluid which is being transported out from the nucleus (such as ejected clouds or cooling gas within the jet)?(b) Is it local gas which is being entrained (heated and accelerated) by the jet and expanding lobes, as they propagate through a relatively dense interstellar medium (ISM)?

We believe that there is fairly strong, but indirect evidence for the latter:

1. The $[\mathrm{N}$ II $]$ lines are far weaker in the extranuclear than in the nuclear gas. This suggests that the extranuclear gas has a different metal abundance from the nuclear gas, as expected for material in the outer regions of a galaxy (e.g., Pagel and Edmunds 1981). As noted above, standard photoionization models using gas with abundances similar to those in the Magellanic Clouds can reproduce the observed spectra in $3 \mathrm{C}$ 277.3. We emphasize that several other galaxies with associated radio continuum and optical line emission exhibit similarly weak extranuclear [N II] lines: NGC 7385 (Simkin, Bicknell, and Bosma 1984; unpublished data from our group), PKS 0349-278 (Danziger et al. 1984; unpublished data from our group), Minkowski’s object (Simkin 1976; van Breugel et al. 1985).

2. The southern radio jet appears to deflect, "light up," and expand near a region of bright optical emission. This suggests that the radio jet has collided with a region of enhanced gas density well outside the nucleus.

3. The radio/optical morphological, kinematic, and spectroscopic properties of the gas in 3C 277.3 are rather similar to those of other objects which have associated radio and optical emission, and for which there does exist other, direct evidence for a relatively dense ISM which may envelop the radio source. Examples are 3C 305 (dust; Heckman et al. 1982), 3C 293 (dust and $\mathrm{H}$ I; van Breugel et al. 1984; Baan and Haschick 1981), 4C
26.42 (cooling X-ray gas; van Breugel, Heckman, and Miley 1984), 4C 29.30 (merger with a disk galaxy; van Breugel, Heckman, and Miley 1985).

4. The line-emitting gas in $3 \mathrm{C} 277.3$, and in other objects with associated radio continuum and optical line emission, is found within $\sim 50 \mathrm{kpc}$ radius of the galaxy nucleus (even when the radio emission extends to much greater distances, such as, for example, in PKS 0349-278). This would be expected if the gas is ambient (local) material associated with the galaxy halo. It would not be expected if the material were nuclear ejecta.

5. To power both the extended optical and radio emission by a jet, the energy-carrying medium would need to travel much faster (at least several thousand kilometers per second) than indicated by the observed emission-line velocities (this is particularly clear in a powerful source like $3 \mathrm{C} 171$; Heckman, van Breugel, and Miley 1984).

We conclude that the evidence drawn from 3C 277.3 itself (points 1 and 2), as well as from other very similar objects (points 3 and 4), and simple energetic considerations (point 5) all imply that the emission-line gas is ambient material which has been energized in some way by the radio plasma.

However, we emphasize that for 3C 277.3 there is no independent evidence for a high ambient gas density. The galaxy appears to be a fairly normal elliptical with no evidence for dust, interaction with a nearby companion, membership in a rich cluster, or X-rays.

The parent galaxy has been classified by Wyndham (1966) as a D2 galaxy, i.e., a galaxy with an elliptical-like nucleus with a fairly extensive optical envelope (following the old classification scheme proposed by Matthews, Morgan, and Schmidt 1964). Also, Branson et al. (1972) note the "diffuse optical envelope parallel to one radio axis." We believe that this classification of $3 \mathrm{C} 277.3$ is now obsolete since we know that this "envelope" is due to the optical line emission, which is brightest along the major radio axis. Deep broad-band optical images would be needed, with the contribution of the optical line emission removed, to determine the possible presence of a large (stellar) halo.

Inspection of the Palomar Sky Survey plates shows that $3 \mathrm{C}$ 277.3 may be at the center of an inconspicuous cluster of galaxies, unclassified by Zwicky and Herzog (1963), behind the eastern outskirts of the Coma Cluster. A spectroscopic survey of these glaxies would be needed to sort out any foreground galaxies belonging to the Coma Cluster before a clusterrichness classification would make much sense. The absence of bright X-ray emission (see below), however, indicates that $3 \mathrm{C}$ 277.3 is unlikely to be a member of a rich cluster.

The upper limit to the X-ray luminosity of 3C 277.3 in the 1-3 keV (Miley et al. 1983) and $0.5-3 \mathrm{keV}$ bands (Fabbiano et al. 1984) is $3.6 \times 10^{42} \mathrm{ergs} \mathrm{s}^{-1}$. Since typical X-ray luminosities of individual elliptical galaxies are $\sim$ a few $\times 10^{41}$ ergs s $^{-1}$ (Forman and Jones 1982; Biermann and Kronberg 1983; Bechtold et al. 1983; Nulsen, Stewart, and Fabian 1983), 3C 277.3 may be quite ordinary in terms of its X-ray luminosity. We will show in $\S$ IVe that the low X-ray luminosity of $3 \mathrm{C}$ 277.3 can then be reconciled with our picture of an optically emitting ambient medium only if the $3 \mathrm{C} 277.3$ halo is strongly inhomogeneous (cloudy).

\section{c) Effects of the Thermal Gas on the Radio Source}

i) Depolarization

Our observations suggest two effects which contribute to the depolarization of the radio emission: $(a)$ Depolarization by an 
irregular Faraday screen in the boundary layers of the radio components, in particular those with associated optical line emission (knots $\mathrm{K}_{1}, \mathrm{~K}_{2}$, northern hot spot). (b) Depolarization by ionized gas which was entrained by the jet and deposited in the southern lobe.

Depolarization by an irregular Faraday screen.--It is almost standard practice in the literature to assume that the observed depolarization in radio sources may be ascribed to Faraday dispersion in a uniformly filled magnetoionic medium. Indeed in 3C 277.3, the various values derived for the electron densities and filling factors of the emission-line gas, together with the minimum-energy values for the magnetic field strength, imply that the observed emission-line gas could possibly depolarize the radio source at $6 \mathrm{~cm}$ if the emission-line clumps were dispersed uniformly throughout the volume of the radio source.

We believe that a more realistic model for the depolarization, at least for the regions with associated optical line emission, is that of an irregular Faraday screen (e.g., Burn 1966; Milne 1980). The preferential occurrence of optical line emission at the edges of the various radio components of $3 \mathrm{C}$ 277.3 suggests that these are surrounded by dense layers of gas which appear optically brighter along the edges because of the longer lines of sight through the gas. The accumulation of this gas in the boundary layers of the source would surround its radio emission with an inhomogeneous Faraday screen, which will depolarize the radio emission if the angular size scale over which the Faraday depth fluctuates is smaller than the telescope beam. This sets a maximum size for the "Faraday cells," while the minimum size is set by the requirement that the cells have adequate Faraday depths. Using the formalism of Burn (1966), these requirements lead to the following loose constraints on the cell size $d_{c}$ (see van Breugel, Heckman, and Miley 1984 for more details in the similar case of 4C 26.42):

$$
\begin{array}{ll}
\text { Hot spot: } & 10^{-6} \mathrm{pc}<d_{c}<300 \mathrm{pc}, \\
\text { Jet knots: } & 10^{-8} \mathrm{pc}<d_{c}<300 \mathrm{pc}, \\
\text { Lobes: } & 10^{-4} \mathrm{pc}<d_{c}<300 \mathrm{pc} .
\end{array}
$$

The presence of irregular Faraday screens may be a relatively general phenomenon, at least on galactic scale sizes. Other objects with line-emitting gas in the boundary layers are for example: 4C 26.42, 4C 29.30, and Minkowski's Object (van Breugel, Heckman, and Miley 1984, 1985; van Breugel et al. 1985). High-resolution rotation measure maps of the jet in NGC 6251 also show evidence for such a screen (Perley, Bridle, and Willis 1984). This may have important implications for the energetics of extragalactic radio sources in general. It is often argued that the radio emission is powered by the kinetic energy of "cold" (thermal) material ejected from the nucleus in the form of a jet. The evidence (if any!) that radio-emitting jets contain appreciable amounts of thermal material rests on observations of Faraday depolarization within the jets, interpreted as arising from the cold jet fluid itself. Instead, the depolarization may be due to the creation of Faraday screens from local material surrounding the outward moving jet. The putative cold jet material itself would then be more tenuous than has been estimated previously. In order that the kinetic energy be supplied at an adequate rate, the jet velocity must then be correspondingly higher.

Depolarization by entrained gas.-The narrow region of depolarization along the jet, southwest of knots $\mathrm{K}_{1}$ and $\mathrm{K}_{2}$, may be caused by (clumpy) gas which has been entrained in the jet from the emission-line region associated with these knots (see $\S \mathrm{IV} d[\mathrm{ii}]$ ). This entrained gas could then act as an irregular Faraday screen for polarized emission behind it. The $\sim 40 \%$ drop in polarization observed across the jet might be explained this way if approximately half of the polarized lobe emission is in front of the depolarizing material in the jet.

The polarization trough at the end of the jet and the rapid decrease in percentage polarization between $6 \mathrm{~cm}$ and $21 \mathrm{~cm}$ in the southwestern half of the southern lobe may be caused by the continuous dumping of this entrained gas. We assume that the jet has expanded and slowed down considerably in the inner region of the southern lobe, and that from there the entrained gas diffuses downstream throughout the southwestern half of the lobe. One would then expect that the gas density is highest in the central regions and decreases outward. This is consistent with the polarization trough and the southwestward increase in percentage polarization at $6 \mathrm{~cm}$ (Fig. 5).

If at any one location in this region the gas is uniformly mixed with the radio-emitting plasma, then the radio emission would depolarize as a result of the effects of internal Faraday dispersion. The Faraday depth of a uniform magnetoionic medium ( $\lambda^{2} \Phi$ in units of radians) is given by Burn (1966):

$$
\lambda^{2} \Phi=1600 n_{e} B_{\|} l \lambda^{2} \mathrm{rad},
$$

where $n_{e}$ is the thermal electron density in $\mathrm{cm}^{-3}, B_{\|}$is the magnetic field strength along the line of sight through the lobe in $\mu \mathrm{G}, l$ is the length of this line of sight in units of $\mathrm{kpc}$, and $\lambda$ is the wavelength in $\mathrm{m}$.

In such a model the radio emission depolarizes rapidly for Faraday depths $\lambda^{2} \Phi \gtrsim \pi$ rad. Assuming a typical value for $B_{\|}$ of $\sim 30 \mu \mathrm{G}$ and $l \approx 12 \mathrm{kpc}$ (Table 4 ), we find that the radio emission is unpolarized at $6 \mathrm{~cm}$ (trough) for $n_{e}>10^{-3} \mathrm{~cm}^{-3}$, and becomes unpolarized between $6 \mathrm{~cm}$ and $21 \mathrm{~cm}$ (southwest of the trough) if $10^{-4} \leq n_{e} \leq 10^{-3} \mathrm{~cm}^{-3}$. These are probably conservative (high) estimates since more realistic models, which include magnetoionic irregularities, including an irregular Faraday screen, would tend to depolarize the source more. In $\S$ IVd(ii) we will argue that the jet entrainment can provide sufficient gas to account for such densities.

*

ii) The Southern Jet: Bending and Decollimation

There are several indications that the southern jet has been deflected in a collision with a massive cloud or cloud complex: (a) The jet is associated with optical line emission $\left(\sim 2 \times 10^{6}\right.$ $M_{\odot}$ of dense gas). (b) The jet and the radio major axis are misaligned by $\sim 30^{\circ}$, and the southern lobe has a bent appearance. The line-emitting gas is located just to the outside of the bend in the radio structure. (c) The two bright knots in the jet near the region of bright emission-line gas may be interpreted as regions of enhanced particle acceleration at the site of a jet-cloud collision (e.g., Blandford and Königl 1979).

Eilek et al. (1984) have suggested that the jet in 3C 465 has been deflected by a collision with a cloud, and we can apply a similar argument concerning the $3 \mathrm{C} 277.3$ jet. The jet thrust $\left(\sim \dot{M}_{j} V_{j}\right)$ will accelerate a cloud of mass $M_{c}$ at a rate $a_{c}$ to a velocity $V_{c}$ in a time $t_{c}$ over a distance $d_{c}$. Then,

$$
M_{c} \sim \frac{\dot{M}_{j} V_{j} t_{c}}{V_{c}} .
$$

We take the jet thrust as equal to the integral of the ram pressure over the jet knots (assuming minimum energy). Then 
$\dot{M}_{j} V_{j} \sim 10^{34}$ dyn. We observe (for the emission-line gas in the $\mathrm{S}$ jet) that $V_{c} \sim 2 \times 10^{7} \mathrm{~cm} \mathrm{~s}^{-1}$. If this cloud has indeed been accelerated by the jet, it can have moved no farther than its current distance from the nucleus $(\sim 10 \mathrm{kpc})$. Then, since $V_{c}=$ $a_{c} t_{c}$ and $d_{c}=\frac{1}{2} a_{c} t_{c}^{2}$, we know that $t_{c}=2 d_{c} / V_{c}<20 \mathrm{kpc} / V_{c}$. Moreover, since the material at the southern extremity of the lobe must also have suffered a similar collision "upstream," $t_{c}>[($ distance from jet to end of lobe $) / c]$. These two constraints on $t_{c}$ yield

$$
10^{8} \mathrm{yr}>t_{c}>10^{5} \mathrm{yr} .
$$

This in turn constrains $M_{c}$ :

$$
7 \times 10^{8} M_{\odot}>M_{c}>7 \times 10^{5} M_{\odot} .
$$

Since we find $M_{c} \sim 2 \times 10^{6} M_{\odot}$ (for the ionized gas at $\sim 1.5 \times 10^{4} \mathrm{~K}$ alone), the cloud would indeed be massive enough to deflect the jet.

The bright radio knots $\left(\mathrm{K}_{1}, \mathrm{~K}_{2}\right)$ are probably evidence of shocks in the jet. We note that they have their sharpest intensity gradients upstream, as would be expected in this case. This is perhaps similar to that observed, in much greater detail, in the jet of M87 (Biretta, Owen, and Hardee 1983). Using the same arguments as these authors and Bridle and Perley (1984), once can show that the material in the jet in 3C 277.3 must be fast $\left(>10^{4} \mathrm{~km} \mathrm{~s}^{-1}\right.$ near knot $\left.\mathrm{K}_{1}\right)$ and hot, as in many other jets (see Bridle and Perley 1984 for a review of jet properties). Thus we emphasize that the velocity of the gas near $\mathrm{K}_{1}(-200 \mathrm{~km}$ $\mathrm{s}^{-1}$ ) is not representative for the material within the jet but presumably refers only to gas entrained in the jet boundaries.

The rapid widening of the jet and its low surface brightness southward of the knots may be due to internal heating by the shocks and rapid slowing down of the jet because of the entrained gas. Detailed theoretical models of the brightness distribution of a deflected and heated fluid jet would be very interesting but are beyond the scope of this paper.

\section{d) Effects of the Radio Source on the External Gas}

\section{i) Excitation: Local Ionization}

The most widely accepted mechanism for the ionization and heating of emission-line gas in active galaxies is the photoionization of the gas by continuum radiation from the nucleus. However, this model is not applicable to $3 \mathrm{C} 277.3$ for several reasons.

Energetics.-Yee and Oke (1978) estimate an optical/ ultraviolet nonthermal continuum luminosity of $\sim 4 \times 10^{42}$ ergs s$^{-1}$ from the nucleus of $3 C 277.3$, while the nuclear X-ray luminosity can likewise be no greater than $3.6 \times 10^{42} \mathrm{ergs} \mathrm{s}^{-1}$ (Miley et al. 1983). Since the jet and the emission-line gas near the $\mathrm{N}$ hot spot could each intercept only about $\sim 2 \%$ of the nuclear continuum (owing to the small solid angles they subtend as seen from the nucleus), each could have total emission-line luminosities of $\sim 10^{41}$ ergs $\mathrm{s}^{-1}$ at most (one to two orders of magnitude below what is observed).

Ionization state.-For $n_{e} \sim 600 \mathrm{~cm}^{-3}$ and a galactocentric distance of $\sim 8.5 \mathrm{kpc}$ for the gas in the $\mathrm{S}$ jet, photoionization models (e.g., Ulrich and Péquignot 1980; Ferland and Netzer 1983) would require a Lyman continuum luminosity of $\sim 10^{46}$ ergs $s^{-1}$ for an ionization parameter high enough to sustain the high degree of ionization seen in the jet gas. This is orders of magnitude in excess of the nuclear nonthermal luminosity (see above).

Instead, a local ionizing source is far more likely in 3C 277.3.
In Miley et al. (1981) we presented observations of polarized optical continuum emission near knot $\mathrm{K}_{1}$ and argued that in the jet, the nonthermal continuum from the jet itself could suffice to photoionize the adjacent gas. Infrared data on knot $\mathrm{K}_{1}$ (Allan 1983), which should be little affected by dust compared with the $B$ flux density reported in Miley et al. (1981), imply a luminosity of $10^{43}$ ergs s$^{-1}$ from 100 to $912 \AA$ in the jet (provided that the infrared-to-ultraviolet continuum has the same slope as the radio-to-infrared continuum). This would indeed be energetically adequate to power the emission-line gas near knot $\mathrm{K}_{1}$.

The situation regarding the local source of ionization for the gas elsewhere in 3C 277.3 remains unclear. Collisional heating by slow shocks, photoionization by radiation produced in fast shocks (Binette, Dopita, and Tuohy 1984), and collisional ionization by relativistic particles (Ferland and Mushotzky 1984) remain viable possibilities.

\section{ii) Gas Entrainment by the Jet}

De Young (1981) has proposed that jets can entrain (heat and accelerate) ambient thermal gas through which they pro-

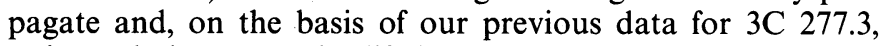
estimated that over the lifetime of the radio source a mass comparable to the mass of the line-emitting gas $\left(\sim 10^{7}-10^{8}\right.$ $M_{\odot}$ ) could well have been entrained. As we have argued in $\S$ $\mathrm{IV} b$, this gas is presumably of local (i.e., nonnuclear) origin, and most of the entrainment probably occurs well outside the nucleus, i.e., near knots $\mathrm{K}_{1}$ and $\mathrm{K}_{2}$. The (suggestive) evidence that entrainment occurs in $3 \mathrm{C} 277.3$ can be summarized as follows.

Morphology.-The gas is observed predominantly along the edges of the radio components (knots, $\mathrm{N}$ lobe, hot spot). At these locations jet plasma can interact intensively with its environment. Sophisticated numerical simulations of axisymmetric jet hydrodynamics (Norman et al. 1982; Norman, Winkler, and Smarr 1983) as well as analogies with laboratory experiments (e.g., De Young 1981) show that the surrounding gas may be entrained in the boundary layers (cocoon) of the jet. It is quite possible that this process also ionizes the gas.

Kinematics.-The strong velocity gradient along the radio axis compared with the weak gradient transverse to it, the particularly strong velocity gradient near the northern hot spot, the asymmetric velocity field, and the broader lines near radio-bright features might all be expected if the gas is being entrained. Alternatives such as cooling jet fluid (nuclear ejecta) or rotating or infalling gas which is merely lit up by jets would be hard-pressed to explain these kinematic peculiarities (which are also observed in other radio sources with emission-line gas).

Note again that the velocities of the line-emitting gas presumably pertain only to the densest phase of the gas entrained in the boundary layers. (The internal jet speeds are probably much higher since the required mass transport rate in the jet required for powering the radio source would be implausibly high at the observed emission-line velocities [Bridle et al. 1981].)

Pressure balance.--If the optical emission-line gas represents material which has cooled isobarically behind shocks driven by the radio source as it entrains the gas, we would expect it to be in rough pressure balance with the radio plasma. This is consistent with the data from near the jet in 3C 277.3.

Depolarization.-In $\S \mathrm{IV} c$ [ii] we proposed that gas entrainment by the jet could account for the peculiar polarization 
structure in the southern lobe. In this section we present arguments which support this.

By analogy to hydrodynamic laboratory experiments, De Young (1981) argued that most of the gas entrainment occurs during the formation of large-scale eddies in the boundary layers of relatively low Mach-number $\left(V_{j} / c_{\text {sound }} \leq 2\right)$ jets. Further evolution of the boundary layer would primarily involve mixing of the entrained material. There may be some evidence that the jet, at least downstream from $\mathrm{K}_{2}$, has a relatively low Mach number: the jet widens with an opening angle of $\sim 40^{\circ}$ (FWHM), which would imply $V_{j} / c_{s} \approx 2.4$ if the jet is freely expanding $(<2.4$ if it is partially confined).

Following De Young (1981), we therefore assume that the entrainment rate $\left(\dot{M}_{\text {ent }} \sim \rho v A ; \rho\right.$ is the density of the entrained gas, $V$ is its velocity, and $A$ is the cross-sectional area where the entrainment takes place) can be estimated by

$$
\dot{M}_{\mathrm{ent}} \approx 60\left(\frac{r_{j}}{\mathrm{kpc}}\right)^{2} \delta n_{0}\left(\frac{c_{s}}{10^{3} \mathrm{~km} \mathrm{~s}^{-1}}\right) M_{\odot} \mathrm{yr}^{-1},
$$

where $r_{j}$ is the jet radius in $\mathrm{kpc}, \delta r_{j}$ is the typical radius of the large-scale eddies $\left(0.1<\delta<1\right.$; see De Young), $n_{0}$ is the average density of the ambient gas in $\mathrm{cm}^{-3}$, and $c_{s}$ is the sound speed in units of $10^{3} \mathrm{~km} \mathrm{~s}^{-1}$.

Assuming that the entrainment by the jet occurs near knots $\mathrm{K}_{1}$ and $\mathrm{K}_{2}$, we take $r_{j}=0.2 \mathrm{kpc}, n_{0}=1 \mathrm{~cm}^{-3}$ on average (using ram pressure arguments similar to those used in $\S$ IVe to derive the external average gas density near the northern hot spot), and a sound speed in the layer of $500 T_{07}^{1 / 2} \mathrm{~km} \mathrm{~s}^{-1}$ $\left(T_{07}=T_{\text {layer }} / 10^{7}\right)$. The entrainment rate is then

$$
\dot{M}_{\text {ent }}=\delta T_{07}^{1 / 2} M_{\odot} \mathrm{yr}^{-1},
$$

and the total amount of gas accumulated at the end of the jet over its lifetime $t_{7}$ (in units of $10^{7} \mathrm{yr}$ ) is

$$
M_{\text {tot }}=\delta T_{07}^{1 / 2} t_{7} 10^{7} M_{\odot} \text {. }
$$

The average gas density in the southern lobe due to this accumulated gas is then

$$
n=M_{\text {tot }} / \mathrm{Vol} \approx 0.4 \delta T_{07}^{1 / 2} t_{7} \mathrm{Vol}_{100}^{-1} \mathrm{~cm}^{-3},
$$

where $\mathrm{Vol}_{100}$ is the volume in units of $100 \mathrm{kpc}^{3}$. A low estimate for $n$ follows if we adopt the following values for the parameters involved.

$\delta=0.1: \quad$ This is probably a lower limit (see De Young).

$T_{07}=1$ : This is a typical temperature for gaseous halos; see, for example, Forman and Jones (1982) and Bechtold et al. (1983).

$t_{7} \approx 0.5: \quad$ This is perhaps the most uncertain parameter. We assume that the age of the source (and jet) is not much less than the current synchrotron lifetime of the lobes $\left(\sim 5 \times 10^{6} \mathrm{yr}\right.$ at $6 \mathrm{~cm}$, Table 4$)$.

$\mathrm{Vol}_{100} \approx 4$ : This is the volume of the SW half of the southern lobe assuming spherical symmetry.

We then find an average gas density of $n \approx 5 \times 10^{-3} \mathrm{~cm}^{-3}$, which (if the gas is ionized) is sufficiently high to depolarize the southern lobe significantly (see $\S$ IV $c[i]$ ).

The band of low brightness in the total-intensity map (Fig. 3), which can be discerned at the same place as the polarization trough, may also be related to the accumulation of entrained gas at the end of the jet. We consider briefly some possible explanations, none of which are entirely satisfactory.

The most simple explanation would be that this band and the concurrent large depolarization are due to anisotropic synchrotron emission, i.e., that a very uniform magnetic field lies along our line of sight through the trough/band. Because of the directivity of the synchrotron emission (maximal perpendicular to magnetic field lines), the total intensity would thus be lowered, while the Faraday depolarization (caused by magnetic fields along the line sight) would be maximized. Such a very uniform field at the end of a jet might be somewhat unexpected, however (if anything, the uniform component of an expanded jet is probably circumferential).

Free-free absorption by ionized gas at $T \sim 10^{4} \mathrm{~K}$ can be ruled out since the emission measure required for optical thickness at $6 \mathrm{~cm}$ would lead to a luminosity which would exceed the observed emission-line luminosity in this region by orders of magnitude. Gas at much greater than $10^{4} \mathrm{~K}$ is even less likely, since the optical depth to free-free absorption goes as $T_{\text {gas }}^{-1.5}$. Also, the spectral index $\alpha_{6}^{21}$ would be expected to vary significantly across the band if the band were due to free-free absorption. This is not observed.

Ionization and radiation losses suffered by relativistic electrons against thermal material would require such high densities $\left(\mathrm{n} \gtrsim 1 \mathrm{~cm}^{-3}\right)$ that only very cool $\left(T \ll 10^{4} \mathrm{~K}\right)$ gas would not violate the X-ray and optical emission-line luminosity constraints. Such neutral gas would not be coupled to the magnetic field, and so the diffusion velocity of the relativistic particles $(\sim$ Alfvén velocity) would be extremely high. The particles would then spend a very short time in the region of enhanced thermal density ( $\ll$ the energy loss time).

\section{e) A Possible Model for the Optical Line Emission Associated with the Northern Hot Spot}

It is generally accepted that the hot spots at the extremities of radio galaxies are caused by jets which impinge on the galactic or intergalactic medium. The jets themselves may or may not be visible at radio wavelengths. The schematic model proposed by Blandford and Rees (1974), i.e., a "working surface" where the jet fluid is decelerated, preceded by a contact discontinuity and a standoff bow shock, has been confirmed and extended in detailed numerical simulations of jets by Norman et al. (1982). The external gas which passes through the bow shock is mixed with the radio-emitting jet fluid and, depending on the details of the models, may flow backward in a cocoon around the jet or form extensive lobes (Norman et al. 1982; Norman, Winkler, and Smarr 1983). In this section we present an interpretation of the morphological relationship of the lineemitting gas to the northern hot spot which uses the above theoretical framework. In particular, we will investigate whether the optical line emission near the hot spot may arise from gas in a circumgalactic halo which encompasses 3C 277.3 and which cools down (to $\sim 10^{4} \mathrm{~K}$ ) after having been compressed, accelerated, and heated by the bow shock.

We identify the steep intensity gradient at the bright northern edge of the hot spot as the current location of the jet's working surface. The northern jet itself remains invisible at radio wavelengths until it reaches the hot spot area (unlike the southern jet, which happened to hit a dense massive cloud near the nucleus).

If the optical line emission is indeed due to compressed halo gas which is cooling in the wake of a bow shock, then, as we now show, the preshock halo gas cannot be uniformly tenuous but must contain denser and cooler clouds. Our argument is primarily based upon the small observed projected distance between the peaks of the optical and radio emission $(\sim 4 \mathrm{kpc})$ and the observed X-ray upper limit for 3C 277.3. 
Let us assume that the standoff shock preceding the end of the northern jet moves through a uniform halo at a velocity which is sufficient to confine the northern radio hot spot by ram pressure (as is strongly suggested by the arrow-shaped large-scale radio structure near the hot spot). Then the shock velocity $\left(V_{s}\right)$ is

$$
V_{s}=\left(\frac{P_{\text {hot spot }}}{\mu m_{\mathrm{H}} n_{\text {halo }}}\right)^{1 / 2},
$$

where $P_{\text {hot spot }}$ is the pressure in the hot spot, $m_{\mathrm{H}}=1.67$ $\times 10^{-24} \mathrm{~g}$, and $\mu=0.6$ (fully ionized gas with cosmic abundances). Taking $P_{\text {hot spot }}=4.5 \times 10^{6} \mathrm{~K} \mathrm{~cm}^{-3}$ (the minimum-energy value, Table 4) then implies

$$
V_{s} \approx 2500\left(n_{\text {halo }} / 0.01\right)^{-1 / 2} \mathrm{~km} \mathrm{~s}^{-1} \text {, }
$$

compared with sound speed

$$
c_{s} \sim 470\left(T_{\text {halo }} / 10^{7} \mathrm{~K}\right)^{1 / 2} \mathrm{~km} \mathrm{~s}^{-1} .
$$

We then assume that the shock is highly supersonic ${ }^{3}$ and calculate the postshock temperature, cooling time, and cooling-layer thickness (e.g., McCray and Snow 1979; McKee and Hollenbach 1980):

$$
\begin{aligned}
T_{s} & =1.4 \times 10^{5}\left(V_{s} / 100 \mathrm{~km} \mathrm{~s}^{-1}\right)^{2} \mathrm{~K}, \\
t_{\text {cool }} & =7.2 \times 10^{3} n_{\mathrm{halo}}^{-1}\left(T_{s} / 10^{6} \mathrm{~K}\right)^{1.6} \mathrm{yr}, \\
l_{\text {cool }} & =6.6 \times 10^{16}\left(V_{s} / 100\right)^{4.2} n_{\text {halo }}^{-1} \mathrm{~cm} .
\end{aligned}
$$

Combining this expression for $l_{\text {cool }}$ with our previous expression for $V_{s}$, we find

$$
l_{\text {cool }} \approx 1800\left(n_{\text {halo }} / 0.01\right)^{-3.1} \mathrm{kpc}
$$

The limits to the X-ray emission of 3C 277.3 (Fabbiano et al. 1984) allow one to estimate a maximum average gas density in the halo if one assumes that the putative halo is spherical, uniform in density (unphysical), and just encompasses the radio source. Adopting a radius of $50 \mathrm{kpc}$ and the cooling functions calculated by Raymond and Smith (1977) in the energy range $0.5-3 \mathrm{keV}$, we find that the upper limit to $L_{x}$ requires the gas density to be $\lesssim 5 \times 10^{-3} \mathrm{~cm}^{-3}$ for $T_{\text {halo }} \approx 10^{7}$ $\mathrm{K}$ (a typical temperature for known gaseous halos; see, for example, Forman and Jones 1982; Bechtold et al. 1983). Thus, the X-ray limits on $n_{\text {halo }}$ imply that $l_{\text {cool }} \geq 125 \mathrm{Mpc}$ ! This is obviously far larger than the observed $4 \mathrm{kpc}$ offset of the brightest radio and emission-line features. For $l_{\text {cool }} \sim 4 \mathrm{kpc}$, $n_{\text {halo }} \sim 7 \times 10^{-2} \mathrm{~cm}^{-3}$ would be required, leading to an X-ray luminosity more than two orders of magnitude larger than the observed upper limit. We conclude, therefore, that the emission-line gas cannot be shocked and cooling halo material, if the halo is uniform.

We therefore consider a model in which the gaseous halo is inhomogeneous and contains regions with enhanced gas densities ("clouds"). The presence of such clouds in the halo is indeed suggested by $(a)$ the clumpy morphology of the optical emission-line gas itself, $(b)$ the high densities observed in the jet knot $\mathrm{K}_{1}\left(n_{e} \approx 600 \mathrm{~cm}^{-3}\right)$, and $(c)$ circumstantial evidence for a $\mathrm{jet} / \mathrm{cloud}$ collision south of the nucleus.

The situation in 3 C 277.3 might then resemble that found in some supernova remnants: the shock preceding the northern

\footnotetext{
${ }^{3}$ We have calculated $l_{\text {cool }}$ for shocks with the appropriate finite Mach numbers using the Rankine-Hugoniot relations. We found values of $l_{\text {cool }}$ only slightly larger than those calculated here.
}

jet travels rapidly through the tenuous intercloud medium in the halo, driving slow radiative shocks into clouds (McKee and Ostriker 1977; McKee, Cowie, and Ostriker 1978). This would also naturally account for the emission-line kinematics: the clouds are accelerated up to $\sim$ three-fourths of the shock speeds in the clouds, and since only slow shocks driven into dense material are radiative $\left(30-300 \mathrm{~km} \mathrm{~s}^{-1}\right.$; see McKee and Hollenbach), one expects to see emission-line velocities of this order, as are observed in 3C 277.3 as well as in other radio galaxies with associated optical line emission.

We can briefly evaluate this picture quantitatively if we assume that the shocks in the clouds are highly supersonic. In that case the clouds (if sufficiently large) will be accelerated to a velocity $V_{c}=\frac{3}{4} V_{\mathrm{sc}}$ ( $V_{c}$ is the cloud velocity; $V_{\mathrm{sc}}$ is the shock velocity in the clouds). We observe $V_{c} \approx 300 \mathrm{~km} \mathrm{~s}^{-1}$ near the hot spot, so that $V_{\mathrm{sc}} \approx 400 \mathrm{~km} \mathrm{~s}^{-1}$. The preshock density $\left(n_{c}\right)$ can then be estimated from the requirement of momentum balance of the fast, intercloud shock $\left(V_{s}, n_{\text {halo }}\right)$ with the slow and radiative cloud shock $\left(V_{\mathrm{sc}}, n_{c}\right)$ :

$$
V_{\mathrm{sc}}=V_{s}\left(\frac{n_{\mathrm{halo}}}{n_{c}}\right)^{1 / 2}
$$

If we again assume ram pressure confinement of the hot spot (predominantly by the hot intercloud halo gas), then

$$
V_{\mathrm{sc}}=250\left(n_{c}\right)^{-1 / 2} \mathrm{~km} \mathrm{~s}^{-1}
$$

so that for $V_{\mathrm{sc}} \approx 400 \mathrm{~km} \mathrm{~s}^{-1}$, we find that the preshock density of the clouds must be typically $n_{c} \approx 0.4 \mathrm{~cm}^{-3}$, and the postshock density, $n_{\mathrm{sc}} \approx 4 n_{\mathrm{c}} \approx 1.6 \mathrm{~cm}^{-3}$. Using these values and the formulae describing the postshock parameters as given above, we find that in the clouds $T_{\mathrm{sc}} \approx 2 \times 10^{6} \mathrm{~K}, t_{\text {cool }} \approx$ $5 \times 10^{4} \mathrm{yr}$.

In $5 \times 10^{4} \mathrm{yr}$ the clouds will have lagged behind the northern edge of the radio hot spot by an amount $\sim\left(V_{s}-V_{\text {sc }}\right) t_{\text {cool }} \approx$ $50\left(V_{s} / 10^{3} \mathrm{~km} \mathrm{~s}^{-1}\right)$ pc (assuming $V_{\text {sc }} \ll V_{s}$, which is appropriate if the cloud/intercloud density contrast is large). This is easily consistent with the proximity of the emission-line and radio features. The above value is actually the minimum distance at which one expects to see optical line emission. The maximum distance depends on the ionization mechanism which keeps the gas at $T \sim 10^{4} \mathrm{~K}$ and on the fact that the clouds will gradually be left behind the hot spot region. Such clouds will then fade from view as they expand (adjusting to the lower pressure of their surroundings) and cool as they separate from their source of ionization. Thus most of the line emission is found primarily near the bright hot spot behind the northern edge, as observed.

If a compressed cloud is sufficiently massive, it would be unstable to gravitational collapse, ultimately resulting in star formation (e.g., De Young 1981; Hardee, Eilek, and Owen 1980; van Breugel et al. 1985). Possibly $\mathrm{SL}_{1}, \mathrm{SL}_{2}$, and $\mathrm{SL}_{3}$ are such $\mathrm{H}$ II regions, since they are not situated near bright radio source components. The weak line emission along the edges of the northern lobe may be weakly compressed cloud material which enters the standoff shock obliquely (at large distances from the shock apex).

Because the clouds are accelerated by the shocks entering them, the kinematics of the thermal gas (velocity $V_{c}$ ) provide only an indirect measure of the kinematics of the radio plasma (velocity $V_{s}$ ):

$$
V_{s} \approx \frac{4}{3} V_{c}\left(\frac{n_{c}}{n_{\text {halo }}}\right)^{1 / 2} \approx 400\left(\frac{n_{c}}{n_{\text {halo }}}\right)^{1 / 2} \mathrm{~km} \mathrm{~s}^{-1}
$$


We do not presently know the cloud density contrast in 3C 277.3. In our own Galaxy's halo it may be $\sim 10^{3}$ (Hartquist 1984). If this is also appropriate to $3 \mathrm{C} 277.3$, then $V_{s} \approx 10^{4} \mathrm{~km}$ $\mathrm{s}^{-1}$ and $n_{\text {halo }} \sim 10^{-4} \mathrm{~cm}^{-3}$. This (intercloud) density is consistent with the X-ray limits.

To summarize, we attribute the great relative strength of the optical emission-line gas in 3C 277.3 to the presence of a cloudy gaseous halo through which shocks are driven by jets. We envisage two important effects of this jet/halo interaction: (a) a local source of ionization is created, and (b) the gas densities in halo clouds are enhanced by their passage through the bow shock and by subsequent cooling (if the cooling is isobaric, gas densities in the clouds may reach $\sim 100 \mathrm{~cm}^{-3}$ near the hot spot, see Table 9).

The existence of an ionization source in bright radio hot spots may be universal in radio galaxies, since 3C 277.3 appears morphologically quite normal. The presence of a dense inhomogeneous external medium, which is needed to actually produce the optical line emission, may however require more special conditions, such as the recent capture of a gas-rich galaxy by the parent galaxy or the accretion of intergalactic gas.

That halo clouds such as those we posit for 3C 277.3 do indeed exist seems fairly well established by the observations of metal absorption-line systems in quasars (see, for example, York 1982; Weymann et al. 1979; Boksenberg et al. 1980; Stocke et al. 1984). However, their properties are poorly known. Bregman (1981) estimates that they should exist out to galactocentric distances of $\sim 10^{2} \mathrm{kpc}$, have gas densities of $10^{0 \pm 1} \mathrm{~cm}^{-3}$, and have temperatures $\lesssim 10^{4} \mathrm{~K}$. Bergeron et al. (1983) have found emission-line gas as far as $150 \mathrm{kpc}$ away from the quasar MR 2251-178, which, like 3C 277.3, is not in a rich cluster, and they estimate average gas densities at $\sim 70$ $\mathrm{kpc}$ of $0.3-0.03 \mathrm{~cm}^{-3}$. The properties of the putative halo clouds in 3C 277.3 (gas densities $\sim 0.4 \mathrm{~cm}^{-3}$, distances $\gtrsim 50$ $\mathrm{kpc}$ from the nucleus) are consistent with these results. Still, it would seem very useful to obtain further information about the environment of 3C 277.3 (more sensitive X-ray observations, deep optical imagery, and the investigation of possible cluster membership).

\section{CONCLUSIONS}

Lest the reader become lost in the forest of data and interpretation we have presented above, we summarize what we believe to be the most important conclusions of this work.

1. There is a convincing morphological association between the extended $\mathrm{H} \alpha$ gas and the radio source (Fig. 4). The brightest $\mathrm{H} \alpha$ peaks lie adjacent to the SE radio knot and NW radio hot spot. A shell-like $\mathrm{H} \alpha$ structure bounds the northern radio lobe. The total amount of observed ionized gas associated with 3C 277.3 might be $\sim 10^{7}-10^{8} M_{\odot}$, and is at least $2 \times 10^{6} M_{\odot}$.

2. Close to the bright southern radio $/ \mathrm{H} \alpha \mathrm{knot}$, the total radio emission bends and widens (Fig. 2), but a narrow "unpolarized jet" remains visible in the percentage polarization maps (Fig. 4). The appearance of the radio source's southern half suggests that the line-emitting gas knot bends and decollimates the radio jet, and is partially entrained in it. The mass of the observed gas in the knot $\left(\sim 2 \times 10^{6} M_{\odot}\right)$ would be sufficient to deflect the jet.

3. In regions of strong $\mathrm{H} \alpha$ emission the radio source is highly depolarized (Fig. 4). Because the $\mathrm{H} \alpha$ emission is generally brightest near the edges of the radio source, this suggests that the radio source is depolarized at these locations by a
Faraday screen associated with the line-emitting gas which surrounds it.

4. The unpolarized jet and the depolarization trough at the end of it (Fig. 4) are probably due to gas which is entrained near the bright radio/optical knot and which is being deposited at the end of the jet in the center of the southern lobe. The presence of a band of low total intensity at this location (Fig. 3) may also be related to this, though the mechanism for producing the band is unclear.

5. There is kinematic evidence for interaction between the relativistic (radio) plasma and thermal (optical line) gas (Table 6 , Fig. 7). The velocity gradient is larger along the radio axis $\left(\sim 500-600 \mathrm{~km} \mathrm{~s}^{-1}\right)$ than perpendicular to it $(\sim 50-100 \mathrm{~km}$ $\left.\mathrm{s}^{-1}\right)$. The emission lines are broader near the bright radio features. The velocity gradient changes sharply near the northern hot spot.

6. The emission-line spectrum shows that (Table 7, Fig. 8) (a) The ionization state of the extended gas is different at different locations and ranges from that of a typical Seyfert 2 to that of a Liner. (b) the ionizing source is of local origin. (c) In the $\mathrm{S}$ jet knots the ionization source is apparently ultraviolet synchrotron radiation from the knots. The local source of the ionization at the other locations remains unclear. $(d)$ The peculiarly weak [N II] lines in the extranuclear spectra can be explained if this gas has chemical abundances similar to the gas in the Magellanic Clouds (cf. Rubin, Ford, and Whitmore 1984).

7. There is evidence that the line-emitting gas has not been transported from the nucleus of the parent galaxy: (a) To power both the extended optical and radio emission by the same jet the energy-carrying medium would need to travel much faster than indicated by the measured gas velocities. (b) Such an origin would not explain the location of the brightest gas at the site of the deflection of the southern jet. (c) The emission-line gas seems to differ in chemical abundances from the nuclear gas (see above).

8. An alternative possibility is that the observed lineemitting gas has condensed from an interstellar or circumgalactic medium in the wake of a shock associated with the outward-moving radio source. This is viable only if the medium is nonuniform (cloudy), since the cooling times are otherwise unacceptably long.

9. Although the kinematics of the observed extranuclear gas are most likely irrelevant to the velocities in the jet, they may well be related indirectly to the outward translation of the radio source.

The authors wish to thank the staffs at NRAO (VLA), KPNO, and Steward Observatory for their assistance in obtaining and reducing the data. We are particularly grateful for the absentee observing service provided by the VLA staff. We acknowledge useful discussions with Drs. D. Young and A. S. Wilson and the constructive comments by the referee Dr. J. Eilek.

In the course of gathering the data and writing the paper during the past several years, the authors have been affiliated with the following institutions, whose hospitality is greatly appreciated: Leiden Observatory (T. H., G. M., W. v. B.), Kitt Peak National Observatory (H. B., G. M., W. v. B.), Steward Observatory (T. H., W. v. B.), National Radio Astronomy Observatory (A. B.), University of Maryland (T. H.), NASA/ Goddard Space Flight Center (T. H.), and Queen's University (A. B.). The final paper was orchestrated while W. v. B. was the 
Bart Bok Fellow at Steward Observatory. T. H. thanks the Alfred P. Sloan Foundation and the National Science Foundation for financial support (grant AST-82-16553), and the Laboratory for Astronomy and Solar Physics at NASA/ Goddard for a NASA/ASEE Summer Faculty Fellowship. G. M. acknowledges partial travel support from NATO grant 828 , and W. v. B. from a grant by the Netherlands Foundation for Pure Research. NRAO is operated by the Associated Universities, Inc., under contract with the National Science Foundation. KPNO is operated by the Association of Universities for Research in Astronomy, Inc., also under contract with the National Science Foundation.

\section{REFERENCES}

Allan, P. 1983, private communication.

Baan, W. A., and Haschick, A. D. 1981, Ap. J. (Letters), 243, L143.

Baldwin, J. A., Phillips, M. M., and Terlevich, R. G. 1981, Pub. A.S.P., 83, 5.

Balick, B., and Heckman, T. M. 1982, Ann. Rev. Astr. Ap., 20, 431.

Bechtold, J., Forman, W., Giacconi, R., Jones, C., Schwarz, J., Tucker, W., and

Van Speybroeck, L. 1983, Ap. J., 265, 26.

Bergeron, J., Boksenberg, A., Dennefeld, M., and Tarenghi, M. 1983, M.N.R.A.S., 202, 125

Biermann, P., and Kronberg, P. K. 1983, Ap. J. (Letters), 268, L69.

Binette, L., Dopita, M. A., and Tuohy, I. R. 1984, preprint.

Biretta, J. A., Owen, F. N., and Hardee, P. E. 1983, Ap. J. (Letters), 274, L27.

Blandford, R. D., and Rees, M. J. 1974, Ap. Letters, 20, 15.

Blandford, R. D., and Königl, A. 1979, M.N.R.A.S., 169, 395

Boksenberg, A., Danziger, I. J., Fosbury, R. A. E., and Goss, W. M. 1980, Ap. J. (Letters), 242, L145.

Branson, N. J. B. A., Elsmore, B., Pooley, G. G., and Ryle, M. 1972, M.N.R.A.S., 156, 377.

Bregman, J. N. 1981, Ap. J., 150, 7.

Bridle, A. H., Fomalont, E. B., Palimaka, J. J., and Willis, A. G. 1981, Ap. J., 248, 499.

Bridle, A. H., and Perley, R. A. 1984, Ann. Rev. Astr. Ap., 22, 319.

Burbidge, G., and Crowne, A. H. 1979, Ap. J. Suppl., 40, 583.

Burn, B. J. 1966, M.N.R.A.S., 133, 67

Cantó, J., Elliott, K. H., Meaburn, J., and Theokas, A. C. 1980, M.N.R.A.S., $193,911$.

Danziger, I. J., Fosbury, R. A. E., Goss, W. M., Bland, J., and Boksenberg, A. 1984, M.N.R.A.S. 298,589

De Young, D. S. 1981, Nature, 293, 43.

Dopita, M. A. 1977, Ap. J. Suppl., 33, 437.

Eilek, J. A., Burns, J. O., Owen, F. N., and O'Dea, C. P. 1984, Ap. J., 278, 37.

Fabbiano, G., Miller, L., Trinchieri, G., Longair, M., and Elvis, M. 1984, $A p$ $J .277,115$.

Ferland, G. J., and Mushotzky, R. F. 1984, Ap. J., 286, 42.

Ferland, G. J., and Netzer, H. 1983, Ap. J., 264, 105.

Forman, W., and Jones, C. 1982, Ann. Rev. Astr. Ap., 20, 547.

Fosbury, R. A. E., Mebold, U., Goss, W. M., and Dopita, M. A. 1978, M.N.R.A.S. 183,549.

Griffin, R. F. 1963, A.J., 68, 421.

Hardee, P. E., Eilek, J. A., and Owen, F. N. 1980, Ap. J., 242, 502.

Hartquist, T. 1984, private communication.

Heckman, T. M. $1980 a$, Astr. Ap., 87, 142.

. $1980 b$, Astr. Ap., 87, 152.

Heckman, T. M., Miley, C. K., Balick, B., van Breugel, W. J. M., and Butcher, H. 1982, Ap. J., 262, 529 .

Heckman, T. M., van Breugel, W. J. M., and Miley, G. K. 1984, Ap. J., 286, 509.

Kellermann, K. I., Pauliny-Toth, I. I. K., and Williams, P. J. S. 1969, Ap. J., 157,1

Koski, A. T. 1978, Ap. J., 223, 56.

Kramer, S. 1984, private communication.
Matthews, T. A., Morgan, W. W., and Schmidt, M., 1964, Ap. J., 140, 35.

McCray, R., and Snow, T. P., Jr. 1979, Ann. Rev. Astr. Ap., 17, 213.

McKee, C. F., Cowie, L. L., and Ostriker, J. P. 1978, Ap. J. (Letters), 219, L23.

McKee, C. F., and Hollenbach, D. J. 1980, Ann. Rev. Astr. Ap., 18, 219.

McKee, C. F., and Ostriker, J. P. 1977, Ap. J., 218, 148.

Miley, G. K. 1983, in Astrophysical Jets, ed. A. Ferrari and A. G. Pacholczyk (Dordrecht: Reidel), p. 99.

Miley, G. K., Heckman, T. M., Butcher, H., and van Breugel, W. J. M. 1981, Ap.J.(Letters), 247, L5.

Miley, G. K., Norman, C., Silk, J., and Fabbiano, G. 1983, Astr. Ap., 122, 330.

Milne, D. K. 1980, Astr. Ap., 81, 293.

Monet, D. G., and Dahn, C. C. 1983, A.J., 88, 1489.

Norman, M. L., Smarr, L., Winkler, K.-H. A., and Smith, M. D. 1982, Astr. Ap., 113, 285.

Norman, M. L., Winkler, K.-H. A., and Smarr, L. 1983, in Astrophysical Jets, ed. A. Ferrari and A. G. Pacholczyk (Dordrecht: Reidel), p. 227.

Nulsen, P. E. J., Stewart, G. C., and Fabian, A. C. 1983, M.N.R.A.S., 208, 185.

Osterbrock, D. E. 1974, Astrophysics of Gaseous Nebulae (San Francisco: Freeman).

Pacholczyk, A. G. 1970, Radio Astrophysics (San Francisco: Freeman).

Pagel, B. E. J., and Edmunds, M. G. 1981, Ann. Rev. Astr. Ap., 19, 77

Perley, R. A., Bridle, A. H., and Willis, A. G. 1984, Ap. J. Suppl., 54, 291.

Raymond, J. C. 1979, Ap. J. Suppl., 39, 1.

Raymond, J. C., and Smith, B. W. 1977, Ap. J. Suppl., 35, 419.

Rubin, V. C., Ford, W. K., Jr., and Whitmore, B. C. 1984, Ap. J. (Letters), 281, L21.

Schmidt, M. 1965, Ap. J., 141, 1.

Simkin, S. M. 1976, Ap. J., 204, 257.

Simkin, S. M., Bicknell, G. V., and Bosma, A. 1984, A p.J., 277, 513.

Stocke, J. T., Liebert, J., Schild, R., Gioia, I. M., and Maccacaro, T. 1984, Ap. $J ., 277,43$.

Thompson, A. R., Clark, B. G., Wade, C. M., and Napier, P. J. 1980, Ap. J. Suppl., 44, 151.

Ulrich, M.-H., and Péquignot, D. 1980, Ap. J., 238, 45.

van Breugel, W. J. M., and Heckman, T. M. 1982, in IAU Symposium 97, Extragalaxtic Radio Sources, ed. D. S. Heeschen and C. M. Wade (Dordrecht: Reidel), p. 61.

van Breugel, W. J. M., Heckman, T. M., Butcher, H., and Miley, G. K. 1984, Ap. J., 277, 82.

van Breugel, W. J. M., Heckman, T. M. and Miley, G. K. 1984, Ap. J., 276, 79.

-1985 , in preparation.

van Breugel, W. J. M., Filippenko, A. V., Heckman, T. M., and Miley, G. K. 1985, Ap. J., in press.

Weymann, R. J., Williams, R. E., Peterson, B. M., and Turnshek, D. A. 1979, Ap. J., 234, 33.

Wyndham, J. D. 1966, Ap.J., 144, 459

Yee, H. K. C., and Oke, J. B. 1978, Ap. J., 226, 753

York, D. G. 1982, Ann. Rev. Astr. Ap., 20, 221.

Zwicky, F., and Herzog, E. 1963, Catalog of Galaxies and Clusters of Galaxies, Vol. 2 (Pasadena: California Institute of Technology).

Note added in proof.-Using recent, more accurate atomic data, Cantó et al. (1980) have slightly revised the relationship between the ratio of [S II] $\lambda 6717$ to [S II] $\lambda 6731$ and the thermal electron density $n_{e}$ as given in Osterbrock (1974). Using this improved relationship, we find $n_{e}=300 \mathrm{~cm}^{-3}$ for the electron density in knot $\mathrm{K}_{1}$, instead of $n_{e}=600 \mathrm{~cm}^{-3}$. Small corrections should therefore be made to other parameters involving the gas density (filling factor, mass of ionized hydrogen, and kinetic energy; see cols. [5]-[7] in Table 8). It does not affect any of our conclusions. We thank Dr. A. V. Filippenko for bringing this to our attention.

Alan BRIDLE: National Radio Astronomy Observatory, Edgemont Road, Charlottesville, VA 22901

HaR vey Butcher: Kapteyn Astronomical Institute, P.O. Box 800, 9700 AV Groningen, The Netherlands

Timothy Heckman: Dept. of Physics and Astronomy, Johns Hopkins University, Baltimore, MD 21218

George MiLeY: Space Telescope Science Institute, Homewood Campus, Baltimore, MD 21218

WIL VAn BREUGEL: University of California, Radio Astronomy Laboratory, 601 Campbell Hall, Berkeley, CA 94720 
PLATE 9

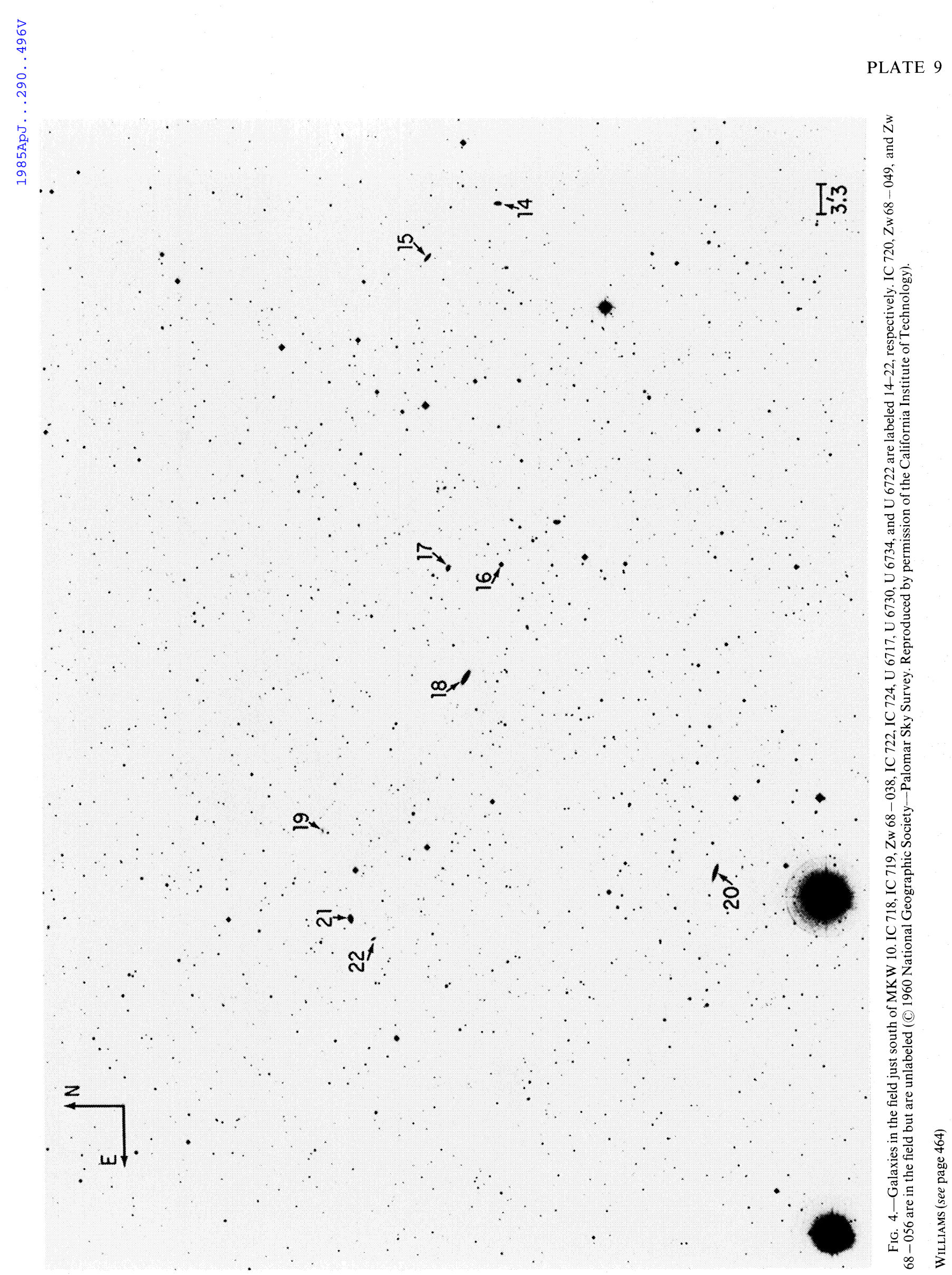




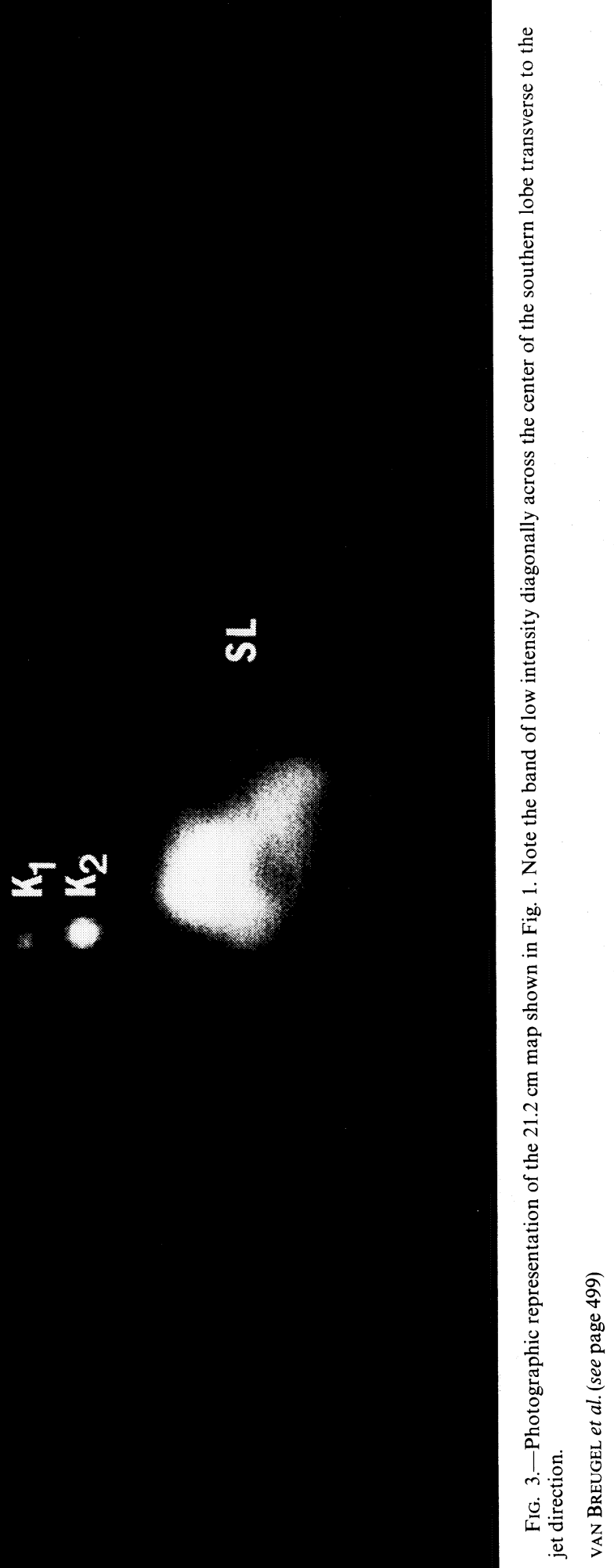

(C) American Astronomical Society - Provided by the NASA Astrophysics Data System 


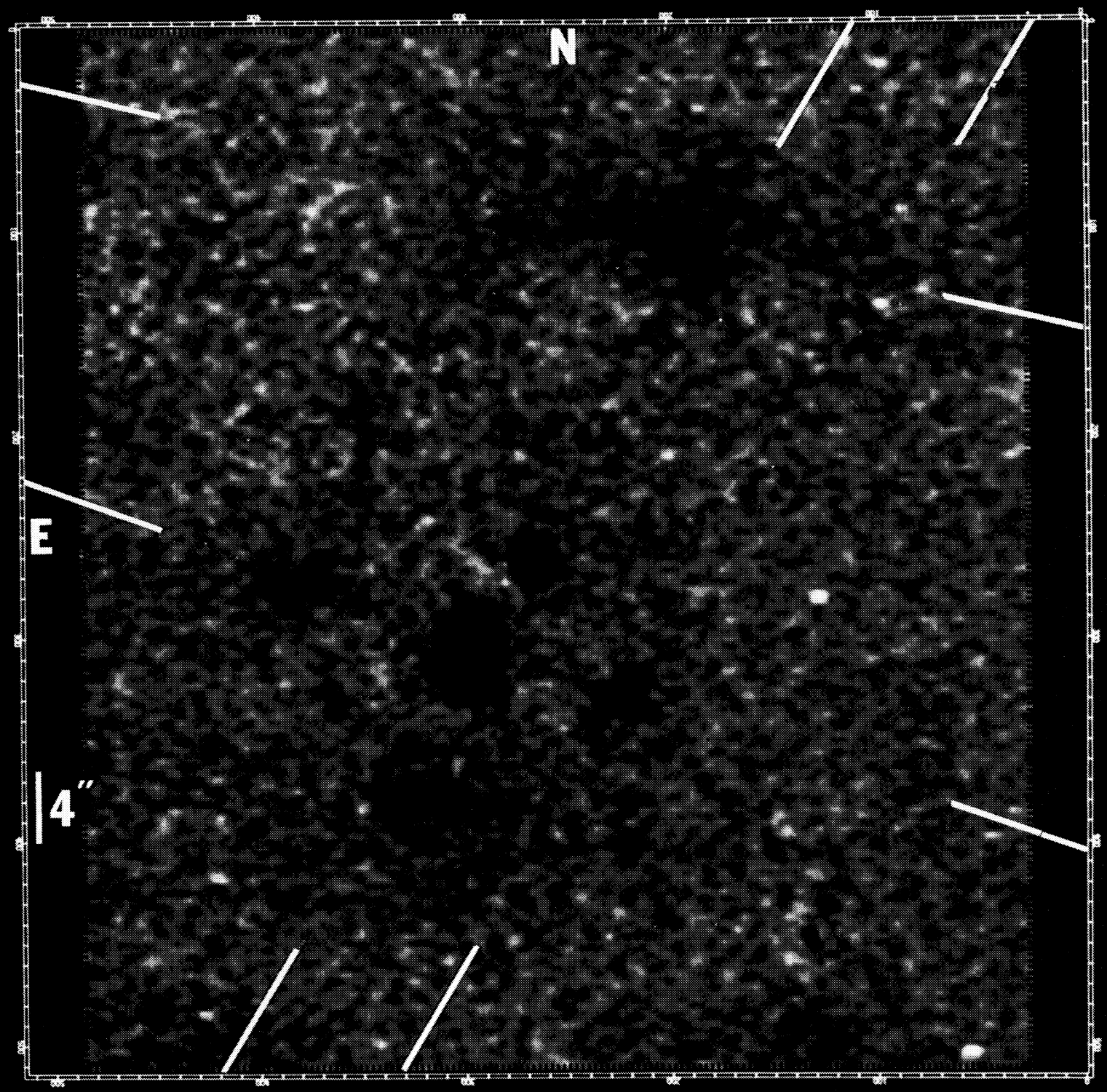

FIG. $6 b .-$ As in Fig. $6 a$ but with the optical continuum subtracted. Various slit position angles are indicated.

VAN BREUGEL et al. (see page 506) 